\title{
Forecasting electricity prices and their volatilities using Unobserved Components
}

\author{
Carolina García-Martos ${ }^{\mathrm{a}, *}$, Julio Rodríguez ${ }^{\mathrm{b}}$, María Jesús Sánchez ${ }^{\mathrm{a}}$ \\ ${ }^{a}$ Escuela Técnica Superior Ingenieros Industriales, Universidad Politécnica de Madrid, Madrid, Spain \\ ${ }^{\mathrm{b}}$ Faculty of Economics and Business Administration, Universidad Autónoma de Madrid, Madrid, Spain
}

Keywords:

Conditional heteroskedasticity

Dynamic factor analysis

Iberian market

Long run

Non-stationary

Short run

\begin{abstract}
A B S T R A C T
The liberalization of electricity markets more than ten years ago in the vast majority of developed countries has introduced the need of modelling and forecasting electricity prices and volatilities, both in the short and long term.

Thus, there is a need of providing methodology that is able to deal with the most important features of electricity price series, which are well known for presenting not only structure in conditional mean but also time-varying conditional variances.

In this work we propose a new model, which allows to extract conditionally heteroskedastic common factors from the vector of electricity prices. These common factors are jointly estimated as well as their relationship with the original vector of series, and the dynamics affecting both their conditional mean and variance. The estimation of the model is carried out under the state-space formulation.

The new model proposed is applied to extract seasonal common dynamic factors as well as common volatility factors for electricity prices and the estimation results are used to forecast electricity prices and their volatilities in the Spanish zone of the Iberian Market.

Several simplified/alternative models are also considered as benchmarks to illustrate that the proposed approach is superior to all of them in terms of explanatory and predictive power.
\end{abstract}

\section{Introduction}

From the early 90 s on, electric power markets of most countries of our socioeconomic environment have been gradually liberalized, abandoning the old regulated-market structures for which the main interest was focused on forecasting demand.

Electricity is exchanged nowadays in competitive markets, as occurs with other commodities, but it presents however some characteristics which make it different, since it cannot be stored and demand needs to be covered immediately. These very peculiar features are responsible for its highly volatile behavior and the difficulty of price forecasting. Thus, there is a need for developing appropriate methodology and models that deal with this problem, since it has a great importance both from the engineering, economic and financial points of view, and affects a strategic sector in the economy of any country. Nowadays, in the context of liberalized markets, modelling electricity prices as well as short and long run forecasting is of great interest, and this has opened a rapidly growing field of research.

\footnotetext{
* Corresponding author at: Escuela Técnica Superior de Ingenieros Industriales, Universidad Politécnica de Madrid. C/ José Gutiérrez Abascal, 2, 28006, Madrid, Spain. Tel.: +34913363148

E-mail addresses: garcia.martos@upm.es (C. García-Martos), jr.puerta@uam.es (J. Rodríguez), mjsan@etsii.upm.es (M.J. Sánchez).
}

In competitive markets, there are several ways to trade electricity:

- Forward markets and options are only well developed in some electricity markets like the EEX in Germany. In others like the Iberian Market (OMIP) more liquidity is needed and this kind of trading is under development.

- By means of bilateral contracts customers and generators may agree to trade a certain amount of power at a certain price. The length of these contracts is usually one year, and an open question is reducing the risk they imply.

- In the pool, both the generating companies and the consumers submit their bids to the market operator, who sorts out the bidding prices for generation or consumption respectively, for each hour of the forthcoming day. Particularly, in the Spanish case, the hourly marginal price is defined as the bid submitted by the last generation unit needed to satisfy the whole demand for the hour considered. This mechanism to obtain what is also known as the market clearing price is shown in rig. 1 . This implies that for each day there is a $24-$ dimensional vector of prices.

The multivariate time series of electricity prices is a high-dimensional panel of series ( 24 or 48 depending on the market considered) which presents jointly some particular empirical features such as seasonality and dependence structure both in the conditional mean and variance 


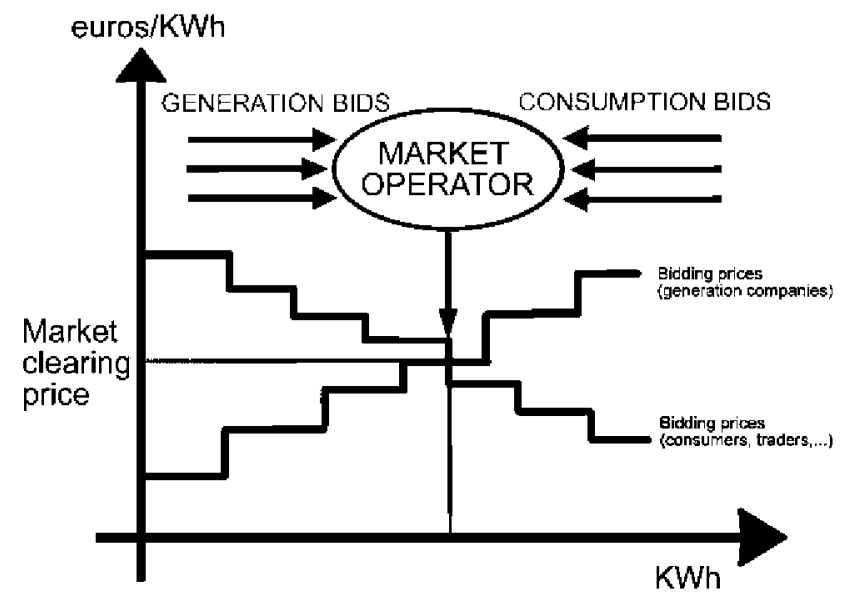

Fig. 1. Market clearing price (Iberian Market).

that make it different to demand series, whose dependence structure do only affect the conditional mean, as well as to financial time series which only present a dependence structure in their conditional variance. Electricity prices present structure both in the conditional mean and variance. The aforementioned characteristics make necessary the development of methodology that is able to deal simultaneously with dimensionality reduction, seasonality and joint structure in the conditional mean and variance, and this is the main purpose of this paper. Our methodological proposal here consists of a novel factor model that allows extracting common factors for the structure in the conditional mean jointly with common volatility factors and is also able to deal with the strong seasonal pattern of the data under study.

It is worth emphasizing that till now, trying to model and forecast electricity prices has implied not only application of previously developed techniques, but also important methodological contributions that can be of application to other data such as macroeconomic or climatic ones.

Several approaches had been considered to model the most important characteristics of electricity prices, such as seasonality, mean reversion or volatility clustering. Bunn (2004) adopted different linear and nonlinear time series techniques. Other contributions of interest in the literature have been made by Lucia and Schwartz (2002) and Knittel and Roberts (2005), who argued for a mean reversion model with deterministic seasonal mean functions and applied it to the Nord Pool and Californian hourly electricity prices. Higgs (2009) focused on modelling volatility inter-relationships between the four Australasian markets by a multivariate GARCH model, after removing the structure in the conditional mean by autoregressive processes.

Focusing on forecasting, both in the short and long run (although there are not many published papers on this difficult issue), a great number of previous works used an univariate approach. On the one hand, computation of short-term forecasts is useful for planning the production of the generation units minimizing costs, and for improving bidding strategies (Conejo et al., 2002, 2004). Some wellknown references in this direction include Nogales et al. (2002), Contreras et al. (2003) and Conejo et al. (2005), who focused on oneday-ahead forecasting for some weeks in the Iberian and Californian markets. We can also mention the paper by Bowden and Payne (2008), who computed short-term forecasts of electricity prices for MISO hubs using ARIMA-EGARCH models and Trapero and Pedregal (2009) provided frequency domain-based estimation methods for unobserved component models, and applied it to forecasting load and prices in the PJM Interconnection.

On the other hand, the disposal of accurate long-run forecasts allows the companies to reduce the risk that is associated to bilateral contracts. They are used to try to dampen the risk derived from the very high volatility of the pool. A precise prediction of the final price allows for a more adequate evaluation of bilateral contracts. This can be done by developing forecasting models with a horizon that covers at least the length of the contract (typically a year). However, there are just a few papers published to date which develop an adequate forecasting model for electricity prices with a horizon larger than a week. Vehvilainen and Pyykkonen (2005) provided medium-term forecasts for monthly electricity prices in the Nord Pool, including exogenous variables such as temperature. Conejo et al. (2010) used the forward prices as an explanatory variable in the long term and estimated the uncertainty associated to pool prices in the medium and long term in the EEX. They used a discretization, and just 48 values were computed for the whole year.

Focusing on alternatives to the univariate approach, Huisman et al. (2007) pointed out that electricity prices should be treated as a panel in which the prices of 24 cross-sectional hours (or 48 in other markets such as the New South Wales in which there are half-hourly prices) vary from day to day. Actually, the multi-equational modelling used by García-Martos et al. (2007, forthcoming), who considered 24 hourly univariate processes (parallel approach) overperforming the results in Nogales et al. (2002), Contreras et al. (2003) and Conejo et al. (2005). The paper by García-Martos et al. (2007) provides a computational experiment to obtain the combination of univariate time series models with the best global performance in the short-run for the Spanish case.

But the multi-equational modelling of the hourly time series developed in García-Martos et al. (2007) could be improved by taking into account the multivariate structure of the data, i.e., fitting a multivariate VARIMA model. But the well-known "curse of dimensionality" arises, since a huge number of parameters must be estimated due to the high dimension of the vector of hourly prices.

To overcome this problem several authors had provided several dimensionality reduction techniques that are applicable to vectors of series that present structure only in the conditional mean, which is not enough to capture all the empirical features of electricity price data. On the one hand, Stock and Watson (2002) focused on reducing dimensionality in panel data used to explain one variable. On the other hand, Peña and Box (1987) proposed a simplifying structure for a vector of time series valid only for the stationary case. Lee and Carter (1992) extended Principal Component Analysis to the case in which the variables are time series, and computed long-run forecasts of mortality and fertility rates by means of extracting a single common factor. More recently, Peña and Poncela $(2004,2006)$ extended the Peña-Box model to the Non-Stationary case. Alonso et al. (2011) proposed the Seasonal Dynamic Factor Analysis, which is able to reduce dimensionality in vectors of series that present a seasonal pattern, they focused on bootstrap-based inference techniques and its application to electricity price data.

On the other hand, as far as vectors of financial data are concerned, usually the conditional variance evolves over time, but the mean process of log-prices can be easily described by a random walk. For this kind of data, several dimensionality reduction techniques had been provided. We can quote the factor GARCH model of Engle (1987), and the static heteroskedastic one-factor model for exchange rate series by Diebold and Nerlove (1989). Harvey et al. (1992) built a modified version of the Kalman Filter for models with unobserved heteroskedastic factors, and they provided an application to the case of a single factor model. This version of the Kalman Filter is also used by King et al. (1994). These models present several drawbacks since they are either static or allowing for just one factor. More recently, Connor et al. (2006) developed a dynamic approximate factor model for extracting common and specific components of dynamic volatility, and García-Ferrer et al. (2011) developed a multivariate generalized independent factor GARCH model. But a common feature of all of them is that they only can deal with series that only present structure in the conditional variance, and this is not enough when dealing with electricity price series. Although some similarities exist between 
electricity markets and financial market operations, electricity prices are more complex due to their strong dependence on load, which is weather and economic-activity dependent. As opposed to financial data, not only the conditional variance of electricity prices evolves over time, but also their conditional mean, and the mean process of electricity log-prices cannot be described simply by a random walk because of its specific characteristics (Escribano et al., 2002 and Bunn and Karakatsani, 2003).

So, apart from the high dimension of the problem, conditional mean and variance must be jointly modelled and there exists a strong seasonal pattern. However, none of the previously mentioned dimensionality reduction techniques are able to deal simultaneously with high-dimensional vectors of series that present both structure in the conditional mean and variance as well as a strong seasonal pattern, which are key empirical features present in panels of hourly electricity prices. That is why an approach used by some authors consists of considering the daily prices so as to have a univariate process instead of a multivariate one, although this involves some loss of information. Koopman et al. (2007) provided novel periodic extensions of dynamic long-memory regression models with autoregressive conditional heteroskedastic errors for the analysis of daily electricity spot prices in several European markets. Higgs and Worthington (2008) modelled daily electricity prices in four Australian electricity markets using three different models: a basic stochastic one, a mean-reverting model and a three regime-switching model.

However, the importance of capturing both the intra-day and inter-day dynamics of electricity prices was highlighted by Smith and Cottet (2006) as well as the presence of seasonality and dependence structure in the conditional mean and variance. They used a Bayesian approach for the estimation of a multivariate stochastic volatility model for the 48 half-hourly time series of prices from the New South Wales market in Australia. They faced the problem of reducing the number of parameters in a completely different way to factor models: assuming the precision matrices of the disturbances of the observation and transition equations to be banded. The model is used to describe the dynamics of electricity prices and their volatilities but out-of-sample forecasts are not computed.

This extensive revision of the literature supports the evidence of the need of introducing a new model that is capable of reducing dimensionality in vectors of series that present structure both in the mean and variance as well as a strong seasonal pattern. The Conditionally Heteroskedastic Seasonal Dynamic Factor Analysis (GARCHSeaDFA) here presented is able to jointly model conditional mean and variance, allowing unobserved common factors extracted from the 24 hourly time series of prices to be conditionally heteroskedastic, following a seasonal VARIMA plus ARCH or GARCH processes. Thus, not only the common structure in mean is extracted, but also (and jointly) the common volatility factors. The GARCH-SeaDFA fills the existent gap in factor models, which are only focused on series that present structure just in the conditional mean or in the conditional variance (financial time series) but not in both of them. In this sense, the GARCH-SeaDFA is a generalization of the models proposed by Alonso et al. (2011) and Harvey et al. (1992). The Homoskedastic SeaDFA is extended to be able to handle vectors of series with common structure in mean and variance and to produce not only price forecasts but also volatility forecasts and forecasting intervals that account for the evolution over time of the conditional variance. The ideas presented in Harvey et al. (1992) for dealing with conditionally heteroskedastic disturbances in state-space models are extended since these authors extracted a single common factor with a much simpler structure governing the evolution over time of the conditional mean (a random walk), so in fact no parameters for the evolution over time of the mean were estimated.

The new model developed is applied not only to modelling electricity prices in the Spanish zone of the Iberian Market (hereafter referred as Spanish Market), but also the overall goal is to compute accurate one-day-ahead and year-ahead forecasts, which are two of the crucial forecasting problems in liberalized electricity markets. Specially, the second one is of great interest since usually the models that perform well in the short run degrade when extending the forecasting horizon.

The rest of the paper is organized as follows. In Section 2 a descriptive analysis of the dataset of prices in the period 1998-2007 in the Spanish Market is provided and linked to the new methodology presented in this paper. In Section 3 the formulation of the GARCHSeaDFA is introduced, as well as its estimation procedure by QuasiMaximum Likelihood (QML). Its application to modelling electricity prices in the Iberian Market during the period 1998-2007, the computation of hourly (one-day-ahead and year-ahead) forecasts for the prices for the whole year 2008 is provided in Section 4, as well as volatility forecasts and prediction intervals that incorporate conditional heteroskedasticity. The comparison with the results obtained with simplified/alternative versions developed by Harvey et al. (1992), García-Martos et al. (2007) and Alonso et al. (2011) are provided. Finally, Section 5 concludes.

\section{Data and descriptive statistics}

In this paper the electricity prices coming from the Spanish zone of the electricity market of the Iberian Peninsula are considered in the period 1998-2008. The data coming from this Market are known for being more difficult to forecast than others (Contreras et al., 2003), because its oligopolist agents often alter the prices for strategic reasons. Thus, checking the performance of the methodology proposed in this paper for this market will be a challenging application.

Firstly, we provide a descriptive analysis of the data in the period 1998-2007. ${ }^{1}$ Fig. 2a shows the boxplot of the hourly prices in the period under study (1998-2007). Variations affecting both the level and variability of the prices depend on the hour considered. These changes are related to the load pattern shown in Fig. $2 \mathrm{~b}$.

According to Fig. 2a, we have selected hours 4, 9, 13 and 21 as representative ones. In Fig. $3 \mathrm{a}$ and $\mathrm{b}$ we show respectively the prices in a subset of the period under study (1998-2005) of the period under study and the conditional variance of the returns for the same four representative hours considered.

Fig. $3 \mathrm{a}$ and Fig. $3 \mathrm{~b}$ illustrate the presence of a common pattern in the evolution over time not only of the conditional mean but also of the conditional variance.

Focusing on Fig. 3a, periods of low and high prices coincide in the four series shown, which are representative of the behavior of the 24 . As far as Fig. 3b is concerned, low, medium and high volatilities occur in the same periods (dates) for all the hours considered. Then, there is also a common pattern in the volatility. This suggests the idea that the evolution over time of the conditional mean and variance could be described by a small number of common factors and common volatility factors respectively, reducing the dimension of the original data by taking advantage of the common features described. Thus, the proposal of a new model that is able to extract seasonal dynamic common factors as well as common volatility factors is needed and meaningful considering the empirical features of the series of electricity prices.

Furthermore, the existence of this common behavior/pattern can be justified bearing in mind the way in which prices are cleared: in liberalized markets, bids for day-ahead delivery of electricity are submitted simultaneously for all hours in the next day (Huisman et al., 2007). Thus, the same common information set is used for quoting all hours in one day. This is an additional evidence from the market operation perspective and also suggests to try to extract unobserved common factors in mean as well as (and jointly) common volatility

\footnotetext{
${ }^{1}$ Last year 2008 would be used in Section 4 to validate the performance of the GARCH-SeaDFA in terms of prediction accuracy, computing out-of-sample forecasts and the corresponding forecasting errors.
} 
a

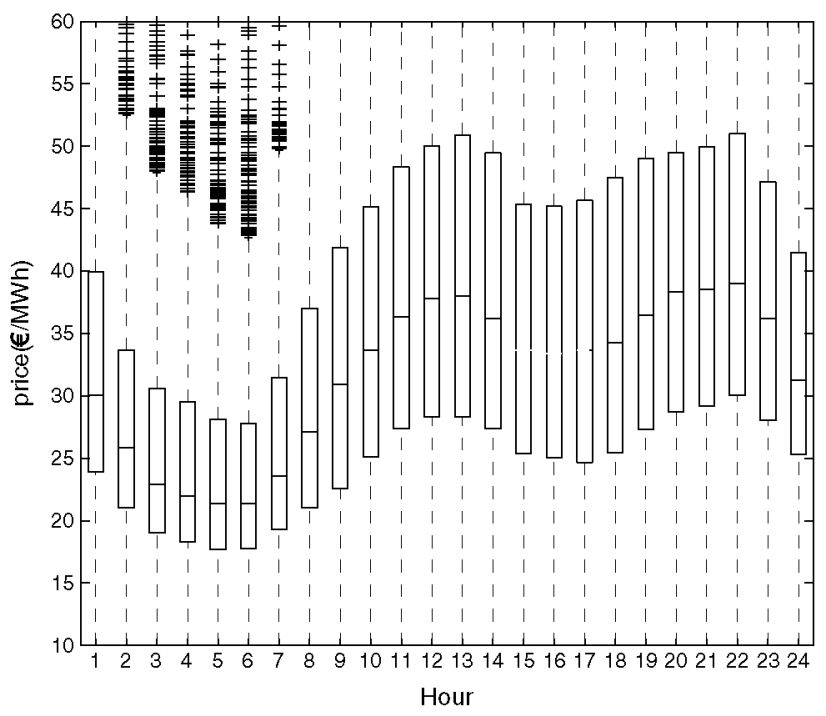

b

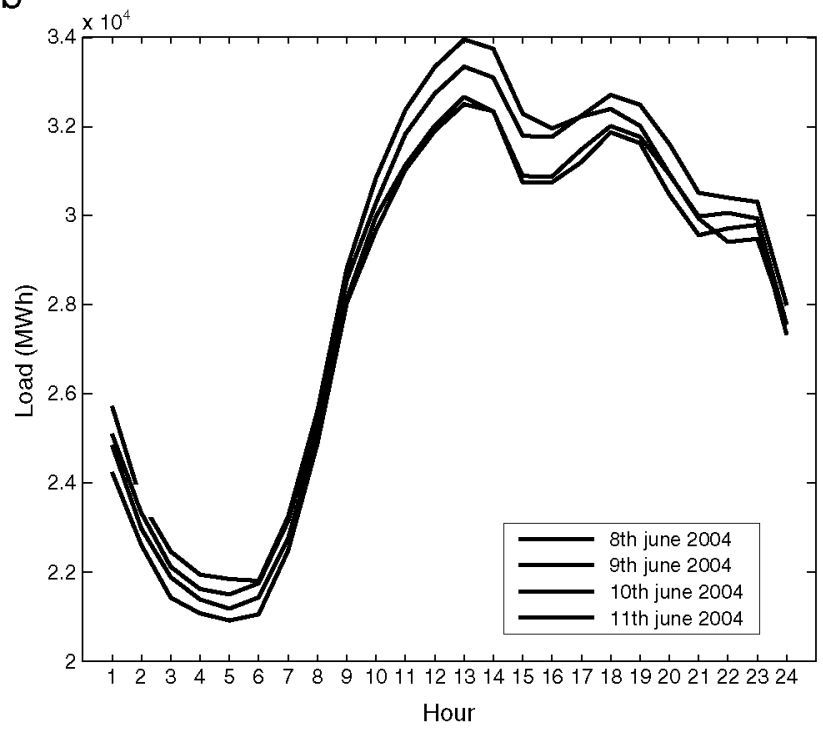

Fig. 2. (a) Boxplot of hourly prices in the period 1998-2007 and (b) examples of hourly load pattern.

factors. A linear combination of these unobserved common factors will give the common part of the original series.

Given that previous works on dynamic factor models are adequate for series with either common pattern affecting the conditional mean or in the conditional variance, but not in both, the Conditionally Heteroskedastic Dynamic Factor Analysis (GARCH-SeaDFA), which fills this gap, is introduced in the next Section.

\section{Formulation of the model}

3.1. Seasonal dynamic factor analysis with homoskedastic disturbances

Alonso et al. (2011) developed the Seasonal Dynamic Factor Analysis (SeaDFA), which is able to extract an $r$-dimensional vector of unobserved seasonal common factors from an $m$-dimensional ob-
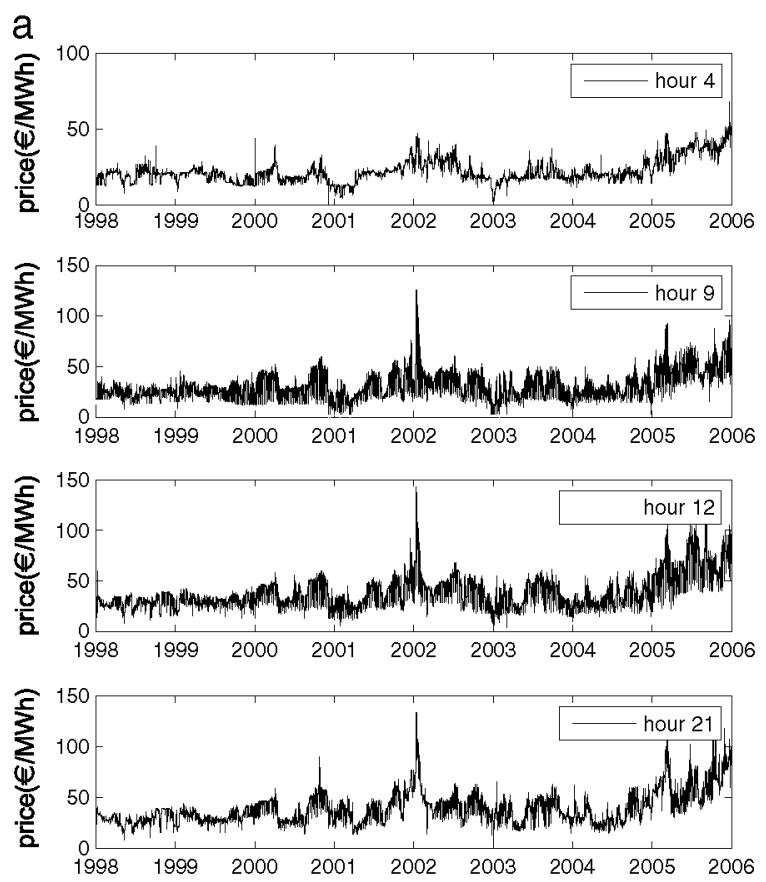

served vector of time series (where $r<m$ ). They assume that vector $\mathbf{y}_{t}$ can be written as a linear combination of the unobserved common factors, $\mathbf{f}_{t}$, plus $\varepsilon_{t}$, to which we will refer from now on as specific components or specific factors:

$\mathrm{y}_{t}=\boldsymbol{\Omega}_{1} \mathrm{f}_{t}+\varepsilon_{t}, \varepsilon_{t} \sim N I I D(0, S)$,

where NIID means Normally, Identically, and Independently Distributed.

Besides, these $r$ common factors are allowed to follow a multiplicative seasonal Vector AutoRegressive Integrated Moving Average, VARIMA $(p, d, q) \times(P, D, Q)_{s}$ model:

$(\mathbf{I}-B)^{d}\left(\mathbf{I}-B^{s}\right)^{D} \boldsymbol{\phi}(B) \boldsymbol{\Phi}\left(B^{s}\right) \mathbf{f}_{t}=\mathrm{c}_{1}+\boldsymbol{\theta}(B) \boldsymbol{\Theta}\left(B^{s}\right) \mathbf{w}_{t}^{1}$,
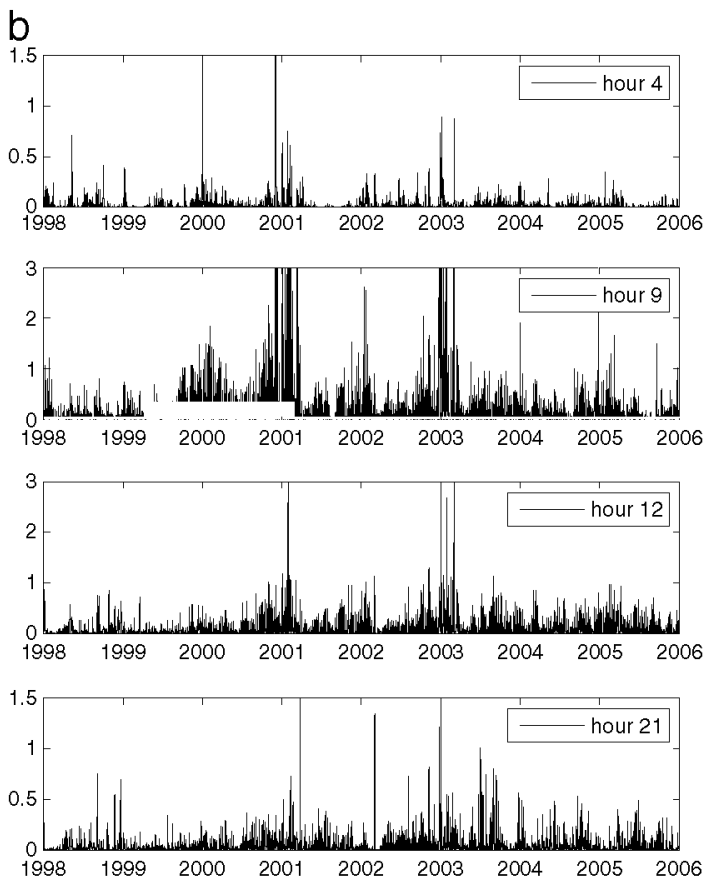

Fig. 3. Selected hourly prices, 1998-2005. Common features in (a) conditional mean and (b) conditional variance. 
where $\mathbf{c}_{1}$ is the $r$-dimensional constant of the model of the common factors, $\boldsymbol{\phi}(B)=\left(\mathbf{I}-\phi_{1} B-\phi_{2} B^{2}-\ldots-\phi_{p} B^{p}\right), \Phi\left(B^{S}\right)=\left(\mathbf{I}-\Phi_{1} B^{s}-\right.$ $\left.\Phi_{2} B^{2 s}-\ldots-\Phi_{P} B^{P s}\right), \boldsymbol{\theta}(B)=\left(\mathbf{I}-\theta_{1} B-\theta_{2} B^{2}-\ldots-\theta_{q} B^{q}\right)$ and $\Theta\left(B^{s}\right)=$ $\left(\mathbf{I}-\boldsymbol{\Theta}_{1} B-\Theta_{2} B^{2 s}-\ldots-\Theta_{Q} B^{Q s}\right)$ are polynomial matrices $r \times r, B$ is the backshift operator such that $B \mathbf{y}_{t}=\mathbf{y}_{t-1}$, the roots of $|\phi(B)|=0$ and $\left|\Phi\left(B^{S}\right)\right|=0$ are on or outside the unit circle, the roots of $|\boldsymbol{\theta}(B)|=0$ and $\left|\Theta\left(B^{S}\right)\right|=0$ are outside the unit circle and $\mathbf{w}_{t}^{1} \sim \mathbf{N}_{r}\left(0, Q_{1}\right)$ is serially uncorrelated for all leads and lags.

The homoskedastic SeaDFA is given by Eqs. (1) and (2), and can be rewritten under the state-space formulation, just reformulating them as an observation and transition Eqs. (3) and (4), respectively:

$\mathrm{y}_{t}=\Omega \mathrm{F}_{t}+\varepsilon_{t}, \varepsilon_{t} \sim N I I D(0, S)$,

$\mathbf{F}_{t}=\mathrm{c}+\Psi \mathbf{F}_{t-1}+\mathbf{w}_{t}, \mathbf{w}_{t} \sim N I I D(0, \mathbf{Q})$.

Concerning the evolution over time of the common factors, in general, taking into account Ansley and Kohn (1986), any multiplicative seasonal VARIMA $(p, d, q) \times(P, D, Q)_{s}$ model as given by Eq. (2), can be easily rewritten as a transition equation like Eq. (4). Thus, the matrices and vectors in Eqs. (3) and (4) are built as follows:

- The transition matrix $\Psi$ would be $b \times b$, where $b=r \cdot b_{1}=r \cdot(s$. $(D+P)+d+p)$,

- $Q$ is also $b$ by $b$ and it is full of zeros except the $r \times r$ upper-left submatrix which is $Q_{1}$,

- $c$ is a column vector of length $b$, whose first $r$ elements are $c_{1}$, and the same holds for the first $r$ elements in $\mathbf{w}_{t}$ and $\mathbf{w}_{t}^{1}$,

- $\boldsymbol{\Omega}$ is $m$ by $b, \boldsymbol{\Omega}=\left[\boldsymbol{\Omega}_{1} \mid 0_{m \times(b-r)}\right]$,

- $F_{t}$ contains the common factors $f_{t}$ and its $\left(b_{1}-1\right)$ lags.

- We also assume that the disturbances $\varepsilon_{t}$ and $\mathrm{w}_{t}$ are uncorrelated for all leads and lags, i.e. $E\left(\mathbf{w}_{t} \varepsilon_{t-h}^{\prime}\right)=0, \forall h$.

Moreover, the model is unidentified under rotations, this problem is solved imposing restrictions like $\mathrm{Q}_{1}=\mathrm{I}$ or $\boldsymbol{\Omega}_{1}^{\prime} \boldsymbol{\Omega}_{1}=\mathrm{I}$, as well as $\omega_{i j}=0$, for $j>i$, where the $\omega_{i j}$ 's are the elements in $\boldsymbol{\Omega}_{1}$. This is not restrictive at all since a rotation can be applied for interpretation purposes when needed. For further details on this identification issue see Geweke and Singleton (1981) and Harvey (1989)

\subsection{Conditionally heteroskedastic seasonal dynamic factor analysis (GARCH-SeaDFA)}

When dealing with vectors of series with a common pattern both in mean and variance, assuming a constant variance for the common factors as explained in the previous subsection does not allow to represent all the features that these datasets present. Considering that we are focusing on electricity prices, allowing the variance of the unobserved common components to evolve over time is more realistic and would represent better the properties of the data under study. Moreover, also the volatility would be predicted and taken into account when building more accurate forecasting intervals, thus accounting for conditional heteroskedasticity enhances the explanatory and predictive power of the model. These intervals can be more relevant than just predicting the price because decisions of all the agents involved are made based on distributions, not only point forecasts, and also the risk assumed is more accurately evaluated. Thus, accounting for conditional heteroskedasticity enhances the explanatory and predictive power of the model.

In this paper, we introduce the possibility of the unobserved common factors having structure both in mean and variance, since we allow for seasonal VARIMA+ARCH/GARCH (Generalized Autorregressive Conditionally Heteroskedastic, Engle, 1982 and Bollerslev, 1986) unobserved common factors. For this purpose the conditionally heteroskedastic disturbances $w_{t}^{*}$ are included in the transition Eq. (4). Thus, the equations of the new model are:

$\mathrm{y}_{t}=\boldsymbol{\Omega} \mathrm{F}_{t}+\boldsymbol{\varepsilon}_{t}, \boldsymbol{\varepsilon}_{\tau} \sim N I I D(0, S)$

$\mathbf{F}_{t}=\mathrm{c}+\boldsymbol{\Psi} \mathbf{F}_{t-1}+\mathbf{w}_{t}^{*}$,

The disturbances $\varepsilon_{t}, \mathrm{w}_{t}^{*}$, which appear in Eqs. (5) and (6) are mutually independent, and according to the description given in Section 3.1 , only the first $r$ components of $\mathbf{w}_{t}^{*}$, i.e.. $\mathbf{w}_{t}^{* 1}$, are different from zero.

As opposed to the aforementioned homoskedastic case, the disturbances of the transition equation follow (in the simplest case) univariate ARCH(1) models:

$\mathbf{w}_{t}^{* 1} \mid I_{t-1} \sim N\left(0_{r \times 1}, \operatorname{diag}\left(\sigma_{1 t}^{2}, o_{2 t}^{2}, \ldots, o_{r t}^{2}\right)\right)=N\left(0_{r \times 1}, Q_{1 t}\right)$,

$\mathbf{w}_{t}^{*} \mid I_{t-1} \sim N\left(\mathbf{o}_{b \times 1}, \operatorname{diag}\left(o_{1 t}^{2}, o_{2 t}^{2}, \ldots, o_{r t}^{2}, \mathbf{o}_{b-r, 1}\right)\right)=N\left(\mathbf{0}_{b \times 1}, \mathbf{Q}_{t}\right)$

$w_{j, t}^{*}=\sigma_{j t} a_{j t}, a_{j t} \sim N I I D(0,1), \sigma_{j t}^{2}=\alpha_{0 j}+\alpha_{j} w_{j, t-1}^{* 2}$, for $j=1, \ldots, r$

where $I_{t-1}$ refers to all the information available at time $t-1$.

It cannot be forgotten that this is a Dynamic Factor Analysis, and that the restrictions provided in the homoskedastic case remain here, since otherwise the model would be unidentified under rotations.

\subsubsection{Quasi-maximum likelihood (QML) estimation and augmented} Kalman Filter

To estimate the parameters involved in this model, we must maximize the log-likelihood function. This function is calculated for models that can be expressed under state-space formulation, which is the case of the GARCH-SeaDFA, using the following expression:

$\log L=-\frac{1}{2} \sum_{t=1}^{T} \log \left((2 \pi)^{n}\left|\Sigma_{t}\right|\right)-\frac{1}{2} \sum_{t=1}^{T} v_{t} \Sigma_{t}^{-1} v_{t}^{\prime}$,

where $v_{t}$ are the innovations and $\Sigma_{t}$ its variance-covariance matrix, and both are obtained as outputs when the Kalman Filter is run. The Kalman Filter equations are given in Appendix A (Further details on these issues can be encountered in Durbin and Koopman, 2001).

When running the Kalman Filter, one of the matrices involved in the calculations is the diagonal variance-covariance matrix of the disturbances of the transition equation, $Q$ in the homoskedastic case and $\mathrm{Q}_{t}=\operatorname{diag}\left(\sigma_{1 t}^{2}, \sigma_{2 t}^{2}, \ldots, \sigma_{r t}^{2}, 0_{b-r, 1}\right)$ in the conditionally heteroskedastic one that we are applying for the GARCH-SeaDFA. Its diagonal elements are constant in the homoskedastic case but evolve over time according to Eq. (7) in the conditionally heteroskedastic one.

Thus, when processing some of the equations of the filter (see Eq. (16) in Appendix A), the computation of $\mathrm{Q}_{t}$, whose diagonal contains $\left(\sigma_{1 t}^{2}, \sigma_{2 t}^{2}, \ldots, \sigma_{r t}^{2}\right)$, is needed, and bearing in mind Eq. (7) the terms $w_{j, t-1}^{* 2}, j=1 \ldots, r$, must be calculated. Given that the $w_{j, t-1}^{*} 2$, $j=1 \ldots, r$ are unobserved, the equations of the Kalman Filter cannot be operated directly. For solving this problem Harvey et al. (1992) proposed replacing $w_{j, t-1}^{*}$ in Eq. (7) by their conditional expectations $E\left(w_{j, t-1}^{* 2} \mid I_{t-1}\right)$ as follows:

$\sigma_{j t}^{2}=\alpha_{0 j}+\alpha_{1 j} w_{j, t-1}^{* 2} \rightarrow \sigma_{j t}^{2}=\alpha_{0 j}+\alpha_{1 j} E\left(w_{j, t-1}^{* 2} \mid I_{t-1}\right)$ for $j=1, \ldots, r$.

And after some minor manipulations it can be easily proved (see Appendix A) that:

$E\left(w_{j, t-1}^{* 2} \mid I_{t-1}\right)=\left(E\left(w_{j, t-1}^{*} \mid I_{t-1}\right)\right)^{2}+E\left[\left(w_{j, t-1}^{*}-E\left(w_{j, t-1}^{*} \mid I_{t-1}\right)\right)^{2}\right](9)$

for $j=1, \ldots, r$,

where the two terms involved are:

- $E\left(w_{j, t-1}^{*} \mid I_{t-1}\right)$ is the expectancy of $w_{j, t-1}^{*}$ conditional on the information available at time $t-1$, and 
- $E\left[\left(w_{j, t-1}^{*}-E\left(w_{j, t-1}^{*} \mid I_{t-1}\right)\right)^{2}\right]$ the precision of the estimate of $w_{j, t-1}^{*}$ with the information available at $t-1$.

If the conditionally heteroskedastic disturbances, $w_{j, t}$, were state variables, then, both $E\left(w_{j, t-1}^{*} \mid I_{t-1}\right)$ and $E\left[\left(w_{j, t-1}^{*}-E\left(w_{j, t-1}^{*} \mid I_{t-1}\right)\right)^{2}\right]$ are direct outputs from the Kalman Filter. Here, we propose estimating the Conditionally Heteroskedastic Seasonal Dynamic Factor Analysis (GARCH-SeaDFA) extending the idea introduced by Harvey et al. (1992), which consists of including the non-zero components of the conditionally heteroskedastic shocks $\mathbf{w}_{\mathrm{t}}^{1 *}$ into the "original" state vector (the common factors $\mathbf{F}_{t}$ ), building the so called "augmented" state vector $\mathbf{F}_{t}^{A}$. For the transition equation, this gives the so called "augmented transition equation", whose matrix form is:

$\mathbf{F}_{t}^{A}=c^{A}+\Psi^{A} \mathbf{F}_{t-1}^{A}+\mathbf{G}^{A} \mathbf{v}_{t}^{A}$,

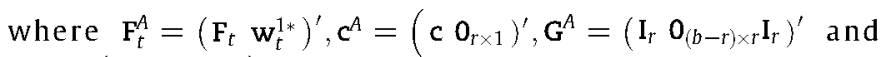
$\Psi^{A}=\left(\begin{array}{cc}\Psi & 0_{b \times r} \\ 0_{r \times b} & 0_{r \times r}\end{array}\right)$.

Since the $w_{t}^{1 *}$ are playing both the roles of state vector and disturbances, $v_{t}^{A}$ denotes $w_{t}^{1 *}$ when it is a disturbance, and the conditional expectation $E\left(v_{t}^{A} \mathbf{v}_{t}^{A_{t}} \mid I_{t-1}\right)=\mathbf{Q}_{t}^{A}$, which is a diagonal matrix whose elements are $\left(\sigma_{1 t}^{2}, \ldots, \sigma_{r t}^{2}\right)$.

Moreover, the observation equation must be replaced by the matrix formulation of its augmented form:

$\mathrm{y}_{t}=\Omega^{A} \mathrm{~F}_{t}^{A}+\varepsilon_{t}$, and $E\left(\varepsilon_{t} \varepsilon_{t}^{\prime}\right)=\mathbf{S}$

where $\boldsymbol{\Omega}^{A}$ is the augmented loading matrix, $\boldsymbol{\Omega}^{A}=\left(\begin{array}{ll}\boldsymbol{\Omega} & 0_{m \tilde{n} r}\end{array}\right)$.

Once we have formulated the Conditionally Heteroskedastic SeaDFA as given by Eqs. (11) and (10), and in order to estimate its parameters, the expression of the log-likelihood in the "augmented" formulation, $\log L^{A}$, must be maximized. It is given by:

$\log L^{A}=-\frac{1}{2} \sum_{t=1}^{T} \log \left((2 \pi)^{\mathrm{n}}\left|\Sigma_{t}^{A}\right|\right)-\frac{1}{2} \sum_{t=1}^{T} v_{t}^{A}\left(\Sigma_{t}^{A}\right)^{-1} v_{t}^{A^{\prime}}$,

where $v_{t}^{A}$ and $\Sigma_{t}^{A}$ are respectively the "augmented" innovations and their variance-covariance matrix. The Kalman Filter can be run for this "augmented" formulation, and the corresponding recursions and details are given in Appendix A.

Finally, a more general model should account for not only transitory disturbances following ARCH processes, but also GARCH ones, since in practice the conditional variance of most of the data can be sufficiently described by a GARCH $(1,1)$ model (for a revision of these models see Tsay, 2005). So, if Eq. (7) is modified in such a way that the disturbances are allowed to follow univariate $\operatorname{GARCH}(1,1)$ processes, the evolution over time of the transitory disturbances is given by:

$$
\begin{aligned}
& \mathbf{w}_{t}^{*} \mid I_{t-1} \sim N\left(0_{r \times 1}, \operatorname{diag}\left(\sigma_{1 t}^{2}, \sigma_{2 t}^{2}, \ldots, \sigma_{r t}^{2}, 0_{(b-r)}\right)\right), \\
& w_{j, t}^{*}=\sigma_{j t} a_{j t}, a_{j t} \sim N I I D(0,1), \sigma_{j t}^{2}=\alpha_{0 j}+\alpha_{j} w_{j, t-1}^{* 2}+\beta_{j} \sigma_{j, t-1}^{2}, \\
& \text { for } j=1, \ldots, r
\end{aligned}
$$

When the values $\sigma_{j t}^{2}$ are needed for the Augmented Filter in the diagonal of $\mathbf{Q}_{t}^{A}$, an additional approximation to the one assumed in Eq. (8) is introduced since there is an extra term, $\beta_{1 j} O_{j, t-1}^{2}$, which would give:

$$
\begin{aligned}
o_{j t}^{2}=\alpha_{0 j} & +\alpha_{1 j} w_{j, t-1}^{* 2}+\beta_{1 j} o_{j, t-1}^{2} \rightarrow o_{j t}^{2}=\alpha_{0 j}+\alpha_{1 j} E\left(w_{j, t-1}^{* 2} \mid I_{t-1}\right) \\
& +\beta_{1 j} E\left(o_{j, t-1}^{2} \mid I_{t-2}\right), j=1, \ldots, r .
\end{aligned}
$$

where $E\left(w_{j, t-1}^{* * 2} \mid I_{t-1}\right)$ is obtained as in (9) and $E\left(\sigma_{j, t-1}^{2} \mid I_{t-2}\right)=\hat{\sigma}_{j, t-1}^{2}$, for $j=1, \ldots, r$, is available from the diagonal of matrix $\mathbf{Q}_{t}^{A}$ estimated in the previous instant of time $t-1$, i.e. $\hat{\mathbf{Q}}_{t-1}^{A}$, whose elements are $\left(\hat{\sigma}_{1, t-1}^{2}, \hat{\sigma}_{2, i-1}^{2}, \ldots, \hat{\sigma}_{r, t-1}^{2}\right)$.

\section{Application to electricity price data}

Here we face two interesting problems to be solved concerning electricity price forecasting. On the one hand, power generation units must be scheduled for the forthcoming day (24-hour ahead) trying to maximize their profits. For this purpose the disposal of accurate oneday-ahead forecasts is crucial for power generators. But also consumers and retailers are interested in having accurate forecasts of electricity prices.

On the other hand, the risk that bilateral contracts imply should be reduced. By means of a bilateral contract consumers agree to purchase some amount of energy at a certain price to a seller for a long period of time, usually a year. But every day the sellers must go to the pool and submit their own bids to buy the energy needed to cover the needs of all their customers. So, there is also a need of computing accurate year-ahead forecasts.

In this section we have also estimated several models to be used as benchmarks, so as to learn about the added explanatory and predictive power of a more sophisticated model such as the GARCH SeaDFA. The models considered for this purpose are the following:

1. The Mixed Model by García-Martos et al. (2007), which is the best general model for the Spanish Market when computing one-dayahead forecasts,

2. The model proposed by Diebold and Nerlove (1989) and Harvey et al. (1992), ${ }^{2}$ who firstly fitted an ARIMA model for capturing the evolution over time of the conditional mean and then estimated a factor model for the common structure in volatility. They dealt with exchange rates data and estimated a single common factor following an $\mathrm{ARCH}(1)$ model after removing the possible structure in the conditional mean using univariate $\operatorname{ARI}(1, p)$ models for the original series.

Since we are modelling the 24-dimensional vector of hourly electricity prices we will use an enhanced version of the Diebold and Nerlove (1989) and Harvey et al. (1992) model (hereafter referred as HRS model), in order to develop a more fair comparison between these models and our GARCH-SeaDFA. This will consist of using a seasonal ARIMA for capturing the dependence structure affecting the conditional mean (instead of just an ARIMA model with regular part) and then two common factors following GARCH $(1,1)$ models will be estimated.

It is worth pointing out that the key difference between these models and the GARCH-SeaDFA is that the common structure is only being captured for the volatility, instead of jointly for the conditional mean and variance, as the GARCH-SeaDFA does. The implementation and computational cost of this model is not much lower than for our GARCH-SeaDFA, since the Augmented Kalman Filter is also used.

3. The Homoskedastic version of the model here proposed, the SeaDFA by Alonso et al. (2011), which allows extracting only the common factors affecting the evolution over time of the conditional mean, and computing forecasts for the price but not for the volatility. This model assumes that the conditional variance of the common factors is constant.

The GARCH-SeaDFA can be considered as a generalization of the second and third models mentioned above, since they can deal respectively with vectors of series with common structure in either

\footnotetext{
${ }^{2}$ This model was firstly considered by Diebold and Nerlove (1989), and the only difference between theirs and the model by Harvey et al. (1992) is that the latter improved the estimation since they proposed using the Augmented Kalman Filter and introduced a "correction term". We have implemented this improved estimation procedure.
} 
the conditional variance or in the conditional mean, but not jointly as in the model here proposed.

An additional simplification which could be considered is the DFA by Peña and Poncela $(2004,2006)$, who did not account for a seasonal pattern in the data under study, so they assumed that the common factors follow a regular VARIMA model instead of a seasonal multiplicative VARIMA. Their assumption simplifies the estimation algorithm significantly, although of course does not allow to capture the common seasonal structure. A detailed comparison between the SeaDFA and the DFA can be encountered in Alonso et al. (2011) where the superiority of the SeaDFA in terms of interpretation and forecasting accuracy is illustrated.

\subsection{Modelling electricity prices and their volatilities in the Spanish zone} of the Iberian Market (1998-2007)

In this subsection, the results obtained when estimating the GARCH-SeaDFA for the prices in Spain are provided. The model proposed and explained in the previous section, is able to obtain from a vector of time series, the common factors for the common structure in conditional mean. Since these factors can present ARCH or GARCH disturbances, common volatility factors are also extracted.

In a first stage, the number of common factors is selected. For the data under study, the $m=24$ hourly time series of electricity prices in the period 1998-2007, $r=2$ common factors are extracted. There is no a specific method for selecting the number of common factors in the presence of conditional heteroskedasticity, but there are some published results focused on the selection of the number of common factors in Dynamic Factor Analysis. Two of them are applied here: the graphical procedure by Forni et al. (2000) whose details can be encountered in Appendix B as well as the a priori test by Peña and Poncela (2006). Thus, when applied to this data, both methodologies provide the same output about the number of common factors, $r=2$.

Considering that electricity prices exhibit a weekly seasonal pattern (there is an instantaneous relationship between load and price and consumption heavily depends on the day of the week, see García-Martos et al., 2007), the order of seasonality is $s=7$. Bearing in mind the importance of seasonality, as mentioned above, this leads us to consider a seasonal unit root in the model for the common factors. This will affect the forecasts incorporating a term for the common expected trend. Concerning the other specifications needed for the model of the common factors, we have selected $p=1$ and $P=1$, since both the common regular and seasonal dynamics must be captured, and simplified versions of the model $(p=1$ and $P=0$ or $p=0$ and $P=1$ ) were not able to. The model chosen for the common factors is a $\operatorname{VARIMA}(1,0,0) \times(1,1,0)_{7}$ with $\operatorname{GARCH}(1,1)$ disturbances, since this model is well known for being general enough to capture the structure in the vast majority of series whose conditional variance evolves over time.

In the particular case under consideration, the GARCH-SeaDFA can be easily written down following the details in Section 3.2 and in Appendix A. It is worth mentioning that in the loading matrix $\omega_{12}=0$, following Harvey (1989) and that $\mathbf{w}_{t}^{1 *}$ has dimension $r=2$ and contains the GARCH disturbances. $c$ is a $b \times 1$ vector whose first $r$ components are $c_{1}$ and $c_{2}$ and the rest of them are zeros. Notice that from the $b \times b$ matrix $\Psi$, only $(p+P) r^{2}=8$ parameters must be estimated (the 2 by 2 matrices of the autoregressive regular and seasonal coefficients). These parameters, or linear and nonlinear relationships between them, appear in the first $r=2$ rows.

Finally, it is worth pointing out that given the complexity of the data, we have fitted a univariate $\operatorname{AR}\left(p_{e}\right)$ model for each of the estimated specific components, $\widehat{\varepsilon}_{t}=\mathrm{y}_{t}-\widehat{\Omega} \widehat{\mathrm{f}}_{t}=\left(\widehat{\varepsilon}_{1, t}, \ldots, \widehat{\varepsilon}_{24, t}\right)$, so as to capture the remaining dynamic structure. The order of the autoregressive model $\left(p_{e}\right)$ for the $e$-th specific component is selected using the Bayesian Information Criterion (BIC). Modelling the univariate remaining structure present in the specific factors was also done by Peña and Poncela (2004) and by Ortega and Poncela (2005), in economic and demographic applications respectively.

Fig. 4a shows the 24-dimensional vector of hourly prices as well as the conditionally heteroskedastic common factors estimated. In Fig. $4 \mathrm{~b}$ the loading matrix that relates observed series of prices and unobserved common factors is provided.

Loads of hourly series in the first factor are all positive and higher in those hours in which the variability is larger according to Fig. $2 a$. Besides, the loads relating observed series and second common factor a

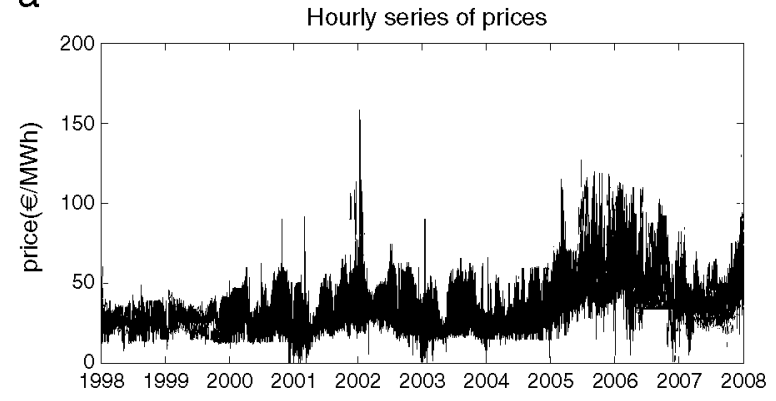

Conditionally Heteroskedastic Common factors

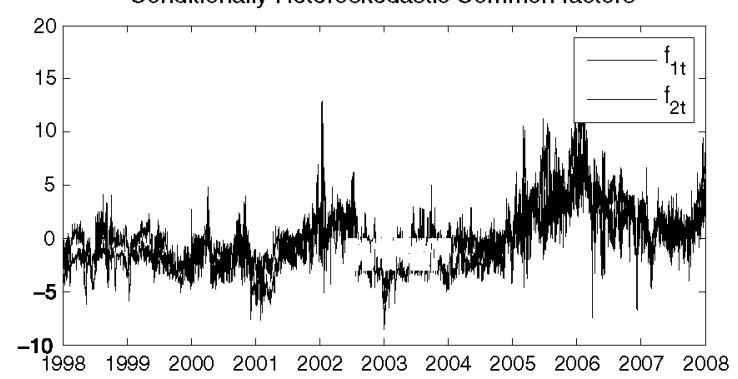

b
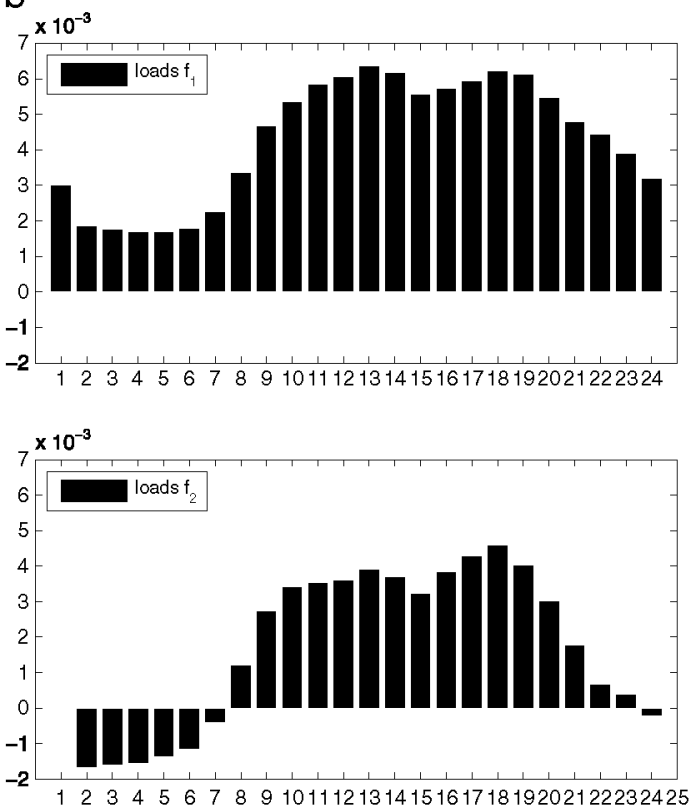

Fig. 4. (a) Vector of hourly prices and unobserved conditionally heteroskedastic common factors (1998-2007) and (b) estimated loads for the common factors. 
are positive for these hours by night and positive for those in the day. There is a clear relationship between the loads estimated and the load pattern given in Fig. 2b.

It must be pointed out that among the 3 benchmarking models considered, only the Homoskedastic SeaDFA is able to extract common and specific features affecting the evolution over time of the conditional mean, as the GARCH-SeaDFA does. Neither the Mixed Model nor the model by Harvey et al. (1992) take into account the multivariate dependence structure in the conditional mean.

Focusing on the comparison between the Homoskedastic and Conditionally Hetereskedastic versions of the SeaDFA, it must be mentioned that with respect to the estimated parameters that affect only the evolution over time of the conditional mean (loads, parameters of the VARIMA model and variances of the specific components), they are negligible. This is sensible and analogous to what happens when estimating an ARIMA and an ARIMA+GARCH to a single univariate time series: the parameters of the ARIMA model slightly change, but additionally we estimate the evolution over time of the conditional variance.

Focusing on electricity price volatilities, the key feature of the GARCH-SeaDFA is that it is able to extract not only dynamic common factors but also (and jointly) common volatility factors. Fig. 5 shows the common volatility factors, $o_{1 t}^{2}$ and $o_{2 t}^{2}$, obtained using the model by the HRS model, those obtained by the GARCH-SeaDFA and the constant variance obtained from the Homoskedastic SeaDFA. The common volatility factors estimated using the GARCH-SeaDFA and the HRS model are rather similar, as well as the estimation of the parameters of the respective $\operatorname{GARCH}(1,1)$ models. From Fig. 5 it is also clear that a weak point of the Homoskedastic SeaDFA is that is not able to model the conditional heteroskedasticity that is present in the common factors (the disturbances of the VARIMA model for the common factors clearly exhibit volatility clusters, and this is shown in Fig. 5 looking at the empirical $\sigma_{1 t}^{2}$ and $\sigma_{2 t}^{2}$ ). Thus, it is not very realistic to estimate a constant variance as the Homoskedastic SeaDFA does.

On the other hand, although the HRS model is able to describe properly the common features in volatility (the results shown in Fig. 5 are very close to those of the GARCH-SeaDFA and both are close to the conditional variance of the conditionally heteroskedastic disturbances, as can be expected), this model is not able to do the same with the common features that affect the conditional mean, since this affects the point forecasts. Furthermore, the computational implementation and cost of the HRS model is not much simpler than for the
GARCH-SeaDFA since it also requires the use of the Augmented Kalman Filter. However, in the next subsections the superiority of the GARCH-SeaDFA when focusing on forecasting is illustrated.

As far as the Mixed Model is concerned, although an advantage is its simplicity and we will see in the following subsection that it looks unbeatable when computing short-run forecasts, it takes into account neither the multivariate structure of the data nor the possibility of modelling the volatility, and its performance when extending the forecasting horizon clearly degrades.

To summarize, the main features related to explanatory power of the models considered are included in Table 1.

\subsection{Long-run forecasting}

The conditionally heteroskedastic dynamic factor model estimated for the data in the period 1998-2007 is used to compute forecasts for the whole year 2008 , which are very useful for the negotiation of bilateral contracts as explained at the beginning of this section. No matter which day in 2008 the hourly forecasts are being computed for, the last data used corresponds to the $31^{\text {st }}$ December 2007.

The accuracy metrics used to check the performance of the model proposed in this paper, are those usually encountered in previous works (Conejo et al., 2005). These accuracy metrics are the MAPE and the MAPE2, where MAPE means Mean Average Percentage Error. These are defined respectively as follows:

MAPE $=\frac{1}{D} \sum_{d=1}^{D}$ emean $_{d}$, MAPE2 $=\frac{1}{D} \sum_{d=1}^{D}$ emedian $_{d}$,

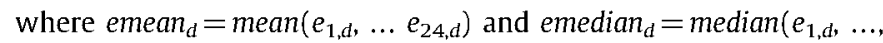
$e_{24, d}$. The percentage error at hour $h$, day $d, e_{h, d}$ is given by $e_{h, d}=\left|p_{h, d}-\hat{p}_{h, d}\right| / p_{h, d}$, where $p_{h, d}$ is the real price at hour $h$ of day $d$, and $\hat{p}_{h, d}$ is its forecast computed using the conditionally heteroskedastic dynamic factor analysis.

In Table 2 the monthly MAPE and MAPE2 are shown for the whole year 2008, using the model estimated with the data from 1998 up to 2007 for the GARCH-SeaDFA, the Mixed Model by García-Martos et al. (2007) and the HRS model (Harvey et al., 1992). Although the Mixed Model overperforms previously published results for the Spanish Market when computing one-day-ahead forecasts, its performance degrades when extending the forecasting horizon: average MAPE and MAPE2 for the whole year 2008 are larger than $70 \%$ and $30 \%$
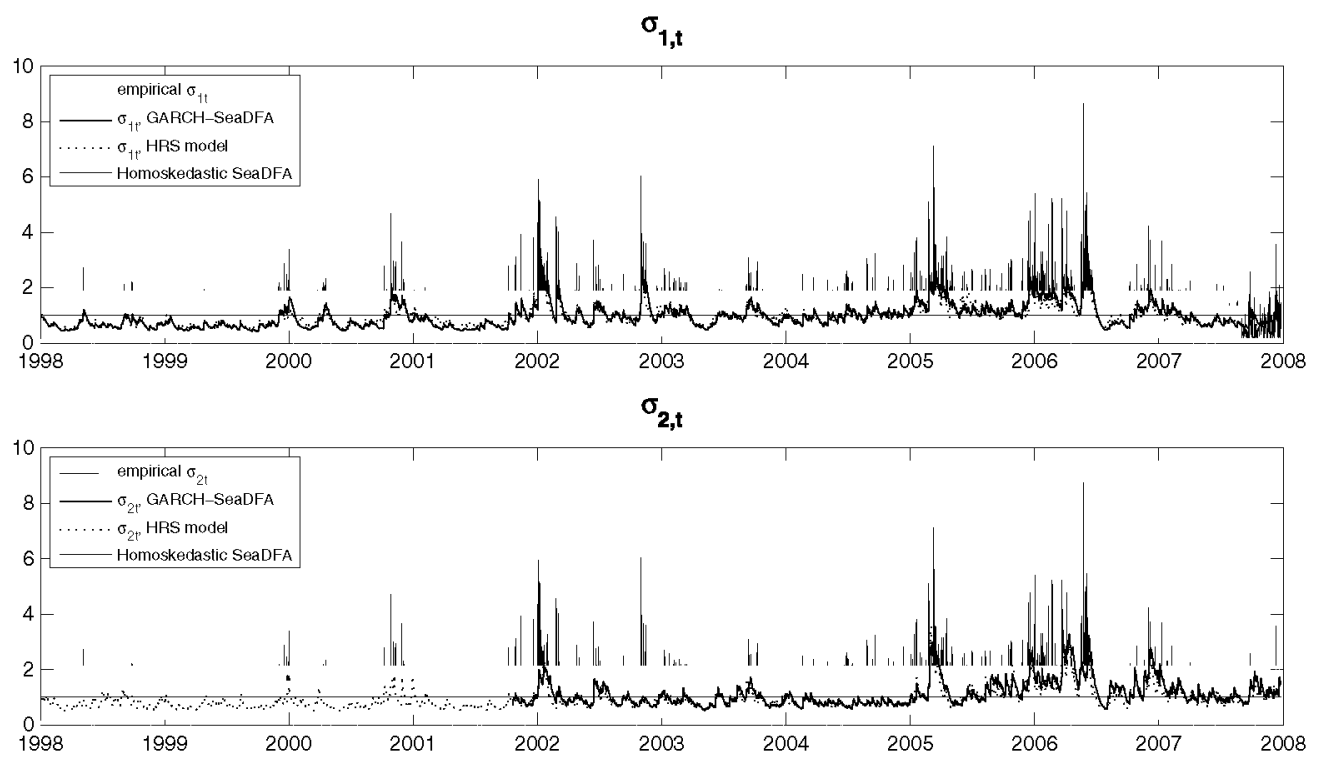

Fig. 5. Common volatility factors estimated using the GARCH-SeaDFA, HRS model and constant variance from the homoskedastic SeaDFA. 
Table 1

Comparison of the features of the models under study: mixed model, HRS model, homoskedastic SeaDFA and GARCH-SeaDFA.

\begin{tabular}{|c|c|c|c|c|}
\hline $\begin{array}{l}\text { Models/ } \\
\text { features }\end{array}$ & $\begin{array}{l}\text { Able to model } \\
\text { volatility of } \\
\text { the data } \\
\text { under study }\end{array}$ & $\begin{array}{l}\text { Account for } \\
\text { multivariate } \\
\text { structure in } \\
\text { the data }\end{array}$ & $\begin{array}{l}\text { Capture } \\
\text { dynamic } \\
\text { common } \\
\text { factors }\end{array}$ & $\begin{array}{l}\text { Capture } \\
\text { common } \\
\text { volatility } \\
\text { factors }\end{array}$ \\
\hline Mixed model & No & No & No & No \\
\hline HRS model & Yes & $\begin{array}{l}\text { Only for the } \\
\text { conditional variance }\end{array}$ & No & Yes \\
\hline SeaDFA & No & $\begin{array}{l}\text { Only for the } \\
\text { conditional mean }\end{array}$ & Yes & No \\
\hline GARCH-SeaDFA & Yes & $\begin{array}{l}\text { Both conditional } \\
\text { mean and variance }\end{array}$ & Yes & Yes \\
\hline
\end{tabular}

respectively, and using the GARCH-SeaDFA these average errors are $16.15 \%$ and $15.22 \%$. As far as the HRS model is concerned, it does not account for the multivariate structure in the conditional mean, and the common volatility factors are extracted to the 24 -dimensional vector of residuals obtained once different univariate seasonal ARIMA models were fitted for each series of hourly prices. We used the BIC criteria to select the most adequate ARIMA model. In most cases using this criteria we selected a model with two unit roots (one regular and one seasonal). As shown in Table 2, where this model is referred as the HRS model (B), this is very convenient in the short run, but not when the forecasting horizon is extended. That is why we also considered what we called the HRS model (A), where we only fixed one seasonal unit root, as we had for the common factors in the GARCH-SeaDFA. By doing so, we think that the comparison between this HRS model referred as HRS model (A) and our GARCH-SeaDFA is more fair than considering the HRS model (B). Anyway, Table 2 illustrates the superiority of the GARCH-SeaDFA, since we got a MAPE of $16.15 \%$, and even with the best HRS model ((A), with a single unit root) the MAPE is $24.63 \%$ (which implies an increase of nearly $50 \%$ in the year-ahead forecasting error with respect to the GARCH-SeaDFA).

\subsection{One-day-ahead forecasting}

The GARCH-SeaDFA model does not only produce accurate forecasts in the long run (year-ahead forecasts provided in previous subsection), but also in the short term. If the model is reestimated every day, and one-day-ahead forecasts are computed for the whole year 2008 , the results are also very good in terms of prediction accuracy.

Usually, in previous works dealing with electricity price forecasting, the last week or the one before last week, depending on which one is a complete week, are used to evaluate the results obtained

Table 2

Year-ahead forecasting errors. Comparison of the GARCH-SeaDFA, Mixed Model by Garcia-Martos et al., 2007 and two different HRS models with different specifications for the conditional mean.

\begin{tabular}{|c|c|c|c|c|c|c|c|c|}
\hline \multirow{2}{*}{$\begin{array}{l}\text { Year } 2008 \\
\text { Month }\end{array}$} & \multicolumn{2}{|c|}{$\begin{array}{l}\text { GARCH- } \\
\text { SeaDFA }\end{array}$} & \multicolumn{2}{|c|}{ HRS model (A) } & \multicolumn{2}{|c|}{ HRS model (B) } & \multicolumn{2}{|c|}{ Mixed Model } \\
\hline & MAPE & MAPE2 & MAPE & MAPE2 & MAPE & MAPE2 & MAPE & MAPE2 \\
\hline January & 20.25 & 19.87 & 23.68 & 23.79 & 14.44 & 12.24 & 14.14 & 12.30 \\
\hline February & 18.00 & 17.91 & 27.78 & 28.52 & 14.22 & 11.26 & 15.49 & 12.53 \\
\hline March & 13.06 & 12.20 & 19.02 & 18.07 & 25.27 & 21.73 & 31.18 & 22.28 \\
\hline April & 13.31 & 12.40 & 16.92 & 15.19 & 36.86 & 31.45 & 46.31 & 28.11 \\
\hline May & 12.82 & 12.20 & 17.17 & 16.03 & 41.62 & 38.98 & 57.93 & 33.25 \\
\hline June & 11.10 & 10.38 & 19.34 & 19.63 & 42.32 & 39.39 & 67.51 & 36.20 \\
\hline July & 16.63 & 15.94 & 29.28 & 30.46 & 32.00 & 27.61 & 63.19 & 28.85 \\
\hline August & 18.30 & 17.73 & 31.39 & 31.98 & 34.15 & 30.48 & 78.17 & 35.08 \\
\hline September & 20.66 & 18.93 & 34.04 & 33.42 & 35.26 & 30.81 & 86.71 & 36.02 \\
\hline October & 17.31 & 15.60 & 30.70 & 29.92 & 43.95 & 38.48 & 106.70 & 40.37 \\
\hline November & 14.98 & 14.17 & 27.81 & 26.96 & 52.50 & 53.22 & 143.78 & 60.78 \\
\hline December & 17.39 & 15.27 & 18.44 & 17.08 & 83.36 & 85.08 & 206.55 & 75.05 \\
\hline Total & 16.15 & 15.22 & 24.63 & 24.25 & 38.00 & 35.06 & 76.47 & 35.07 \\
\hline
\end{tabular}

Table 3

Day-ahead forecasting errors for the third week of each month in 2008, comparison of the GARCH-SeaDFA and the Mixed Model by García-Martos et al. (2007).

\begin{tabular}{lll}
\hline Week & $\begin{array}{l}\text { MAPE } \\
\text { (GARCH-SeaDFA) }\end{array}$ & $\begin{array}{l}\text { MAPE } \\
\text { Mixed Model }\end{array}$ \\
\hline 21-27 January 2008 & 6.59 & 8.30 \\
18-24 February 2008 & 9.13 & 7.59 \\
24-30 March 2008 & 6.60 & 9.86 \\
21-27 April 2008 & 6.72 & 5.14 \\
19-25 May 2008 & 6.72 & 6.22 \\
23-29 June 2008 & 5.40 & 5.18 \\
21-27 July 2008 & 5.76 & 5.31 \\
25-31 August 2008 & 6.77 & 5.52 \\
22-28 September 2008 & 6.41 & 4.33 \\
20-26 October 2008 & 6.65 & 9.98 \\
17-23 November 2008 & 7.35 & 7.80 \\
22-28 December 2008 & 5.66 & 15.61 \\
Average & & \\
\hline
\end{tabular}

(Conejo et al., 2005). In Table 3, the MAPE for these selected weeks is provided, as well as those obtained using the Mixed Model by GarcíaMartos et al. (2007), since according to Table 2 it is the best one among the 3 benchmarking models considered when focusing on the short run. Furthermore, the Mixed Model was specifically designed for one-day-ahead forecasting, and it is the model with the best performance for the Spanish Market, overperforming the proposals by Nogales et al. (2002) or Contreras et al. (2003). In the Mixed Model intervention analysis is performed to deal with outliers. However, in the GARCH-SeaDFA model presented in this paper, we are not incorporating intervention analysis to the QML estimation procedure described in Section 3. This could be done as further research, since it exceeds the goals of this paper.

Anyway, the weekly errors for the weeks considered in Table 3 are similar or slightly better using the GARCH-SeaDFA provided here, compared to the Mixed Model. Nevertheless, for long-run forecasting the forecasts produced with the Mixed Model give much larger errors as pointed out in the previous Subsection.

\subsection{Volatility forecasting and prediction intervals for electricity prices}

A very important feature of electricity prices is that they present conditional heteroskedasticity. The GARCH-SeaDFA is able to extract common volatility factors, as shown in Section 4.1, and it is also possible to compute one-day-ahead forecasts for the common volatility factors. Volatility forecasts are interesting per se, but a very relevant issue is that they can be used to compute prediction intervals for the prices that take into account the fact that electricity price data present structure both in conditional mean and variance.

Given Eqs. (5) and (6) where $\mathrm{w}_{t}^{*} \mid I_{t-1} \sim N\left(0, \mathrm{Q}_{t}\right)$, prediction intervals for electricity prices that incorporate the evolution over time of the common volatility features are given by:

$$
\left(\hat{\boldsymbol{y}}_{T+k \mid T}-z_{1-\alpha / 2} \sqrt{\Sigma_{T+k \mid T}}, \hat{\boldsymbol{y}}_{T+k \mid T}+z_{1-\alpha / 2} \sqrt{\Sigma_{T+k \mid T}}\right)
$$

where $\hat{\boldsymbol{y}}_{T+k \mid T}$ is the point prediction and $\boldsymbol{\Sigma}_{T+k \mid T}$ its MSE (see Rodríguez and Ruiz, 2009 for details). Volatility forecasts $\hat{\sigma}_{1 T+k \mid T}^{2}$ and $\hat{\sigma}_{2 T+k \mid T}^{2}$ are involved in the calculation of $\Sigma_{T+k \mid T}$, so differences appear between the Homoskedastic and Conditionally Heteroskedastic models, which are illustrated for the third week in November 2008 in Fig. $6^{3}$. In Eq. (14), $\hat{\boldsymbol{y}}_{T+k \mid T}$ and $\Sigma_{T+k \mid T}$ are supposed to be computed assuming known parameters. In practice we replace them by consistent estimates obtained by the QML procedure described in Section 3.2 .

\footnotetext{
${ }^{3}$ In general the third week of each month and more specifically the third one in November are used by different authors to check the performance of different forecasting methodologies applied to electricity markets (Conejo et al., 2005; Nogales et al., 2002).
} 


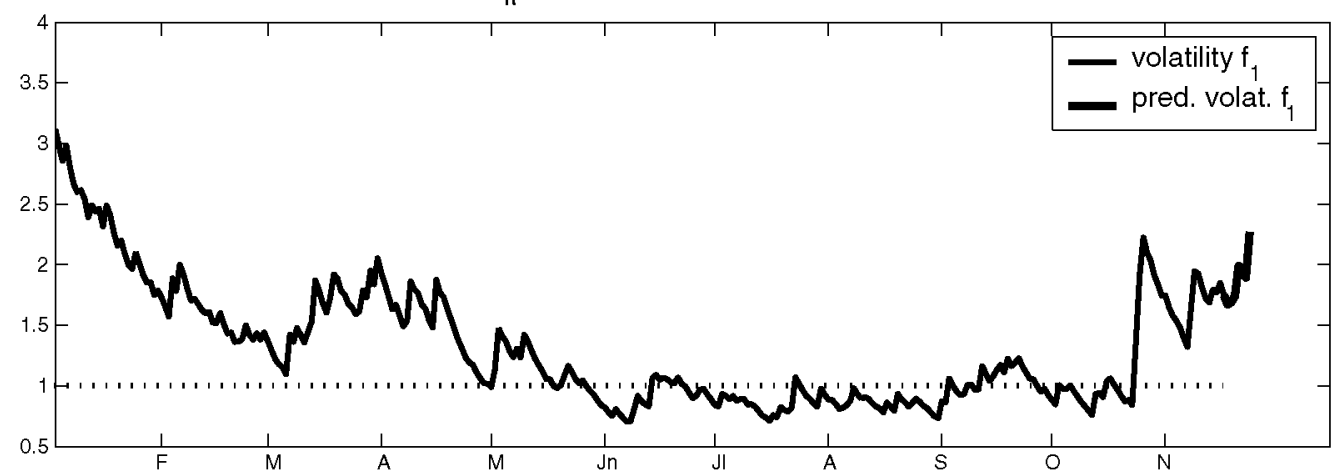

$\sigma_{2 t}$ (1st January - 23rd November 2008)

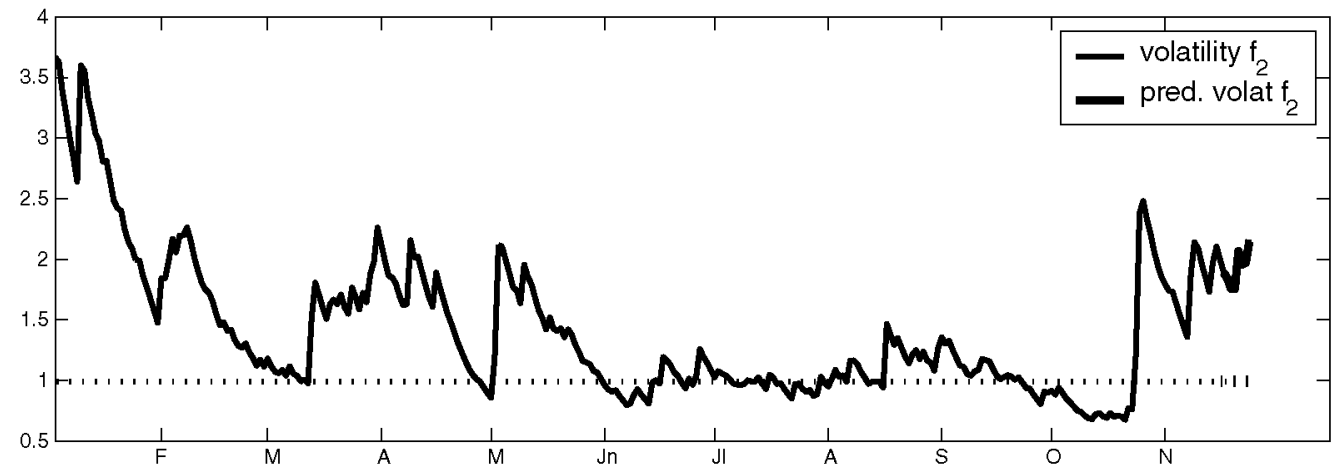

Fig. 6. Volatility estimates 1 Jan-16 November 2008 and volatility forecasts: $17-23$, November 2008 . (Dotted line corresponds to the homoskedastic case).

Fig. 6 shows the volatility estimated for the first common factors using the GARCH-SeaDFA, only the part corresponding to year 2008 up to 16th November and forecasts for the volatility in the third week in November 2008 . The comparisons of the estimated volatility for the whole period under study for several models considered are shown in Fig. 5. Moreover, volatility forecasts can be more clearly shown if the period of time plotted in the same graph is not so long. As an important conclusion, it can be stated that in low volatile periods, assuming a constant variance as the Mixed Model and Homoskedastic SeaDFA could over-estimate the variance, so the prediction intervals could be wider than true ones. As opposed to this, in highly volatile periods, assuming a constant variance could under-estimate the width of forecasting intervals. As shown in Fig. 5 the volatility estimates obtained with the GARCH-SeaDFA and the HRS model are similar (which is sensible, as explained in Section 4.1).

Focusing on the forecasting intervals, and the comparison between all the models considered, although the width of the forecasting intervals would be really similar when using the GARCH-SeaDFA and the HRS model, the coverages are clearly better using the GARCHSeaDFA, given that the point forecasts $\hat{\boldsymbol{y}}_{T+k \mid T}$ in which these intervals are centered are much more accurate when using the GARCH-SeaDFA according to Table 2 . It is important to emphasize that the characterization in each hour is very important, and it can be more relevant that just predicting the point price (decisions are made based on distributions, not just on point forecasts).

Once the superiority of the GARCH-SeaDFA has been detailed in this and the previous subsection, in terms of explanatory and predictive power, we provide in Fig. 7 real prices, point forecasts for the prices and prediction intervals for the third week in November 2008. They have been obtained with the new model introduced in this paper, the GARCH-SeaDFA. These intervals incorporate conditional heteroskedasticity present in common factors.

Allowing the variance of the common factors to evolve over time permits to correctly estimate (not under or over estimating) the width of forecasting intervals, which is very important for the correct risk management that all the agents involved in the operation of electric markets assume.

\section{Conclusions}

This paper faces the important problem of forecasting electricity prices and their volatilities in liberalized markets. Moreover, computing short-term forecasts and specially those of long-run forecasting is still an open problem, and the methodology here introduced is motivated by these important issues.

Electricity prices present structure both in the conditional mean and conditional variance, so it is necessary to develop methodology that is able to capture jointly both dynamics. Although electricity prices share some features with data coming from financial markets,

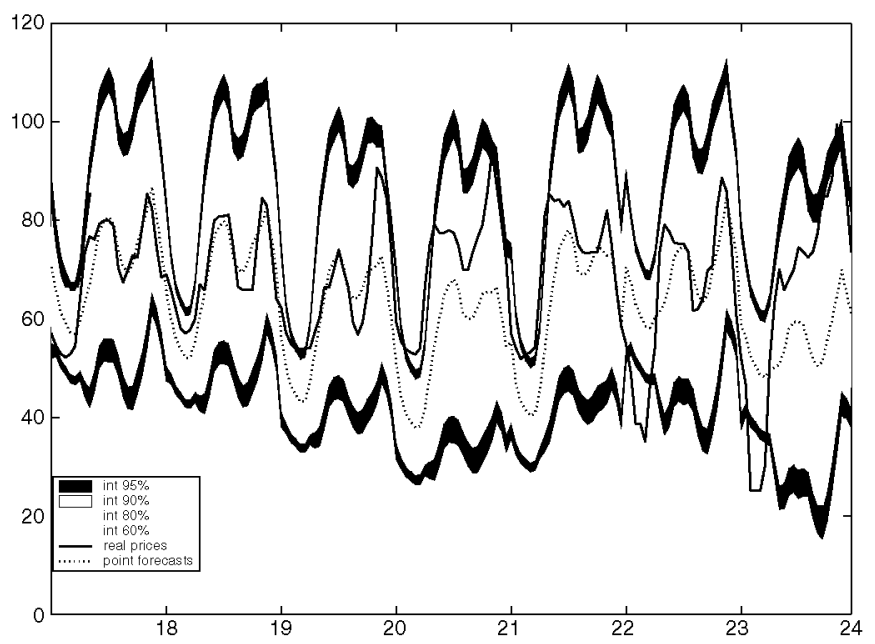

Fig. 7. Point forecasts and forecasting intervals in the week 17 th-23rd November 2008 
also the structure in the conditional mean must be modelled jointly with that in conditional variance. The high dimension of the problem, 24 hourly time series, justifies the application of dimensionality reduction techniques. Moreover, the extraction of common factors from the vector of hourly series of prices is justified bearing in mind the operation in electricity market, since the same information set is used for quoting all hours of the forthcoming day (Huisman et al., 2007).

However, the existent dimensionality reduction techniques for multivariate time series were only of application to vectors of series with either structure in the conditional mean or variance, but not in both. In this paper we develop the Conditionally Heteroskedastic Seasonal Dynamic Factor Analysis, allowing for unobservable common factors that exhibit conditional heteroskedasticity.

The methodology developed by Harvey et al. (1992) and Alonso et al. (2011) is generalized in a more complete model that is able to deal with the case in which the common factors present conditional heteroskedasticity. This new model proposed is able to extract conditionally heteroskedastic common factors from any high-dimensional vector of time series.

Detailed numerical results are provided for the Spanish Market, focusing on modelling electricity prices and volatilities and producing forecasts both in the long and short run. The prices and volatilities have been modelled for the period 1998-2007 using the new GARCHSeaDFA model. Moreover, concerning forecasting, the results for all the hourly prices in the year 2008 are calculated and validated, both for the short and long term.

A detailed comparative study for the new model proposed and the three benchmarking ones is carried out in terms of explanatory power for the dynamics of prices and their volatilities as well as related to the accuracy of long and short term forecasts and volatilities and forecasting intervals. As a result of these comparisons, the superiority of the proposed method becomes of great importance.

The new model has a very good performance in terms of prediction accuracy both for the short and long term. Particularly, long-run forecasting is a difficult issue, and there are no many published works about it. Moreover, results concerning volatility forecasting are also included, as well as forecasting intervals that incorporate the evolution of volatility over time.

The methods here proposed, in which the common trend of a vector of series is captured both for the conditional mean and variance, could be of application in the field of macroeconomic data.

\section{Acknowledgements}

The authors would like to thank Andrés M. Alonso and JoséMira for their help and comments as well as financial support by Projects ECO2009-10287 and MTM2009-12419, Ministerio de Ciencia e Innovación, Spain and SAS-IIF Grant to Support Research on Principles of Forecasting in the year 2007.

\section{Appendix A. Standard and Augmented Kalman Filter}

When dealing with a model that can be expressed under its statespace formulation, as it is the case of the Homoskedastic SeaDFA (Alonso et al., 2011) described by Eqs. (1) and (2) or the GARCHSeaDFA given by Eqs. (5) and (6), the Kalman Filter (KF) recursions allow to compute the latent variables at time $t=t$ conditioning on the information available till $t-1$ or $t$, say $\mathbf{F}_{t \mid t-1}=E\left[\mathbf{F}_{t} \mid\left(\mathbf{y}_{1}, \mathbf{y}_{2}, \ldots \mathbf{y}_{t-1}\right)^{\prime}\right]$ and $\mathrm{F}_{t \mid t}=E\left[\mathrm{~F}_{t} \mid\left(\mathrm{y}_{1}, \mathrm{y}_{2}, \ldots \mathrm{y}_{t}\right)^{\prime}\right]$, respectively. The filter provides the computation of the precision of the state or latent variables. Particularly, we will use the notation $P_{t_{1}, t_{2} \mid z}=E\left[\left(\mathbf{F}_{t_{1}}-\mathbf{F}_{t_{1} \mid z}\right)\left(\mathrm{F}_{t_{2}}-\mathbf{F}_{t_{2} \mid z}\right)^{\prime}\right]$, and the simplified one $P_{t \mid z}$ when $t_{1}=t_{2}=t$. In the case $z<t$ the problem is forecasting, if $z=t$ we have a filtering problem and although the smoother is not presented here it holds when $z>t \mathrm{KF}$ recursions are given by Eqs. (15) to (20).
In the homoskedastic model described in Subsection 3.1 we can directly proceed with this standard formulation of the KF, just replacing $Q_{t}$ by $Q$, and maximize the log-likelihood to estimate the parameters of the model. However, in the new model presented in this paper we are allowing for a time-varying variance-covariance matrix of the disturbances of the transition equation, $Q_{t}$, and we have considered the particular case in which $\mathrm{Q}_{t}=\operatorname{diag}\left(o_{1 t}^{2}, o_{2 t}^{2}, \ldots, o_{r t}^{2}, 0_{(b-r)}\right)$. A particular case (the simplest one) of the GARCH-SeaDFA considers ARCH(1) disturbances, where $\sigma_{j t}^{2}=\alpha_{0 j}+\alpha_{1 j} w_{j, t-1}^{* 2}, j=1, \ldots, r$. The KF recursions for this model are the following:

$\mathbf{F}_{t \mid t-1}=\mathrm{c}+\Psi \mathbf{F}_{t-1 \mid t-1}$,

$P_{t \mid t-1}=\boldsymbol{\Psi} P_{t-1 \mid t-1}^{\prime} \boldsymbol{\Psi}^{\prime}+\mathbf{Q}_{t}=\boldsymbol{\Psi} P_{t-1 \mid t-1}^{\prime} \boldsymbol{\Psi}^{\prime}+\operatorname{diag}\left(\sigma_{1 t}^{2}, \sigma_{2 t}^{2}, \ldots, \sigma_{t t}^{2}, \mathbf{0}_{(b-r)}\right)$,

$v_{t}=\mathrm{y}_{t}-\boldsymbol{\Omega F} \mathrm{F}_{t \mid t-1}$

$\Sigma_{t}=\boldsymbol{\Omega} P_{t \mid t-1} \boldsymbol{\Omega}^{\prime}+S$

$\mathbf{F}_{t \mid t}=\mathbf{F}_{t \mid t-1}+P_{t \mid t-1} \boldsymbol{\Omega}^{\prime} \Sigma_{t}^{-1} v_{t}$

$P_{t \mid t}=P_{t \mid t-1}-P_{t \mid t-1} \Omega^{\prime} \Sigma_{t}^{-1} \Omega P_{t \mid t-1}$

But these equations of the Standard KF cannot be operated directly in our conditionally heteroskedastic case, given that, for the computation of the $\sigma_{j t}^{2}$ in the diagonal of $Q_{t}$, the squared unobserved disturbances $\left(w_{j t}^{*}\right)^{2}$ are needed. Since $w_{j t}^{*}$ are unobserved, we need to apply and extend the ideas developed by Harvey et al. (1992), who approximated the $\left(w_{j, t-1}^{*}\right)^{2}$ by their conditional expectations, $\left(w_{j, t-1}^{*}\right)^{2} \rightarrow E\left[\left(w_{j, t-1}^{*}\right)^{2} \mid I_{t-1}\right]$. Additionally, using the following trick:

$w_{j, t-1}^{*}=E\left(w_{j, t-1}^{*} \mid I_{t-1}\right)+\left(w_{j, t-1}^{*}-E\left(w_{j, t-1}^{*} \mid I_{t-1}\right)\right)$

the calculation of the conditional expectation $E\left[\left(w_{j, t}^{*}\right)^{2} \mid I_{t-1}\right]$ gives:

$E\left[\left(w_{j t}^{*}\right)^{2} \mid I_{t-1}\right]=\left(E\left(w_{j, t-1}^{*} \mid I_{t-1}\right)\right)^{2}+E\left[\left(w_{j, t-1}^{*}-E\left(w_{j, t-1}^{*} \mid I_{t-1}\right)\right)^{2}\right]$,

since the cross-product is zero. The first term in Eq. (22), $E\left(w_{j, t-1}^{*} \mid I_{t-1}\right)$, $j=1, \ldots, r$ is given by the last $r$ elements in $\mathrm{F}_{t-1 \mid i-1}^{A}=E\left(\mathrm{~F}_{t-1}^{A} \mid I_{t-1}\right)$, which is a direct output from the Augmented KF. Additionally, the second additive term in Eq. (22), i.e. $E\left[\left(w_{j, t-1}^{*}-E\left(w_{j, t-1}^{*} \mid I_{t-1}\right)\right)^{2}\right], j=1, \ldots, r$, is given by the elements $(b+1)$ to $(b+r)$ in the diagonal of $\mathrm{P}_{t-1 \mid t-1}^{A}=E\left[\left(\mathrm{~F}_{t-1}^{A}-E\left(\mathrm{~F}_{t-1}^{A} \mid I_{t-1}\right)\right)^{2}\right]$, which is also a direct output obtained from the Augmented KF (the first $r$ elements in $P_{t-1 \mid t-1}^{A}$ correspond to $E\left[\left(F_{k, t-1}^{*}-E\left(F_{k, t-1}^{*} \mid I_{t-1}\right)\right)^{2}\right], k=1, \ldots, r$.

Thus, the elements in the diagonal of $\mathrm{Q}_{t}^{A}$, i.e., $o_{j t}^{2}, j=1, \ldots, r$, are calculated using Eq. (22) and bearing in mind the specification of an $\mathrm{ARCH}(1)$ model given in Eq. (7):

$$
\begin{aligned}
o_{j t}^{2} & =\alpha_{0 j}+\alpha_{1 j} w_{j, t-1}^{* 2} \rightarrow \alpha_{0 j}+\alpha_{1 j} E\left(w_{j, t-1}^{* 2} \mid I_{t-1}\right) \\
& =\alpha_{0 j}+\alpha_{1 j}\left\{\left(E\left(w_{j, t-1}^{*} \mid I_{t-1}\right)\right)^{2}+E\left[\left(w_{j, t-1}^{*}-E\left(w_{j, t-1}^{*} \mid I_{t-1}\right)\right)^{2}\right]\right\} .
\end{aligned}
$$

The two terms needed are directly obtained from the Augmented $\mathrm{KF}$ when the conditionally heteroskedastic disturbances are included 
as state variables as follows, which affects both the observation and transition equation:

$$
\begin{aligned}
& \left(\begin{array}{c}
\mathbf{F}_{t} \\
\mathbf{w}_{t}^{1 *}
\end{array}\right)=\left(\begin{array}{c}
\mathbf{c} \\
0_{r \times 1}
\end{array}\right)+\left(\begin{array}{ll}
\boldsymbol{\Psi}_{b \times b} & 0_{b \times r} \\
0_{r \times b} & 0_{r \times r}
\end{array}\right)\left(\begin{array}{c}
\mathbf{F}_{t-1} \\
\mathbf{w}_{t-1}^{1 *}
\end{array}\right)+\left(\begin{array}{c}
\mathbf{w}_{t}^{*} \\
\mathbf{w}_{t}^{1 *}
\end{array}\right) \\
& \left(\begin{array}{c}
\mathbf{F}_{t} \\
\mathbf{w}_{t}^{1 *}
\end{array}\right)=\left(\begin{array}{c}
c \\
0_{r \times 1}
\end{array}\right)+\left(\begin{array}{ll}
\boldsymbol{\Psi}_{b \times b} & 0_{b \times r} \\
0_{r \times b} & 0_{r \times r}
\end{array}\right)\left(\begin{array}{c}
\mathbf{F}_{t-1} \\
\mathbf{w}_{t-1}^{1 *}
\end{array}\right)+\left(\begin{array}{c}
I_{r} \\
0_{(b-r) \times r} \\
I_{r}
\end{array}\right) \mathbf{w}_{t}^{1 *},
\end{aligned}
$$

$\mathrm{y}_{t}=\left(\begin{array}{ll}\boldsymbol{\Omega} & 0_{m \times r}\end{array}\right)\left(\begin{array}{c}\mathbf{F}_{t} \\ \mathbf{w}_{t}^{1 *}\end{array}\right)+\varepsilon_{t}$,

Eqs. (24) and (25) are the expanded versions of the matrix form of the augmented formulation of the model given by Eqs. (10) and (11), respectively.

For these equations of the model in the augmented formulation the Augmented KF recursions that should be applied are Eqs. (26) to (31):

$\mathbf{F}_{t \mid t-1}^{A}=c^{A}+\Psi^{A} F_{t-1 \mid t-1}^{A}$

$P_{t \mid t-1}^{A}=\Psi^{A} P_{t-1 \mid t-1}^{A} \Psi^{A \prime}+G^{A} Q_{t}^{A} G^{A t}$

$v_{t}^{A}=\mathrm{y}_{t}-\boldsymbol{\Omega}^{A} \mathbf{F}_{t \mid t-1}^{A}$

$\Sigma_{t}^{A}=\Omega^{A} P_{t \mid t-1}^{A} \Omega^{A \prime}+S$

$\mathbf{F}_{t \mid t}^{A}=\mathbf{F}_{t \mid t-1}^{A}+\left(P_{t \mid t-1}^{A}\right)^{\prime} \boldsymbol{\Omega}^{A \prime}\left(\Sigma_{t}^{A}\right)^{-1} v_{t}^{A}$

$P_{t \mid t}^{A}=P_{t \mid t-1}^{A}-\left(P_{t \mid t-1}^{A}\right)^{\prime}\left(\boldsymbol{\Omega}^{A}\right)^{\prime}\left(\Sigma_{t}^{A}\right)^{-1} \boldsymbol{\Omega}^{A} P_{t \mid t-1}^{A}$

The augmented innovations, $v_{t}^{A}$, as well as their variancecovariance matrix, $\Sigma_{t}^{A}$, are direct outputs of the filter needed to build the log-likelihood function described in Section 3.2, and whose maximization will lead to the estimates of all the parameters in the model.

Appendix B. Illustration of the application of the Forni et al. (2000) test for the selection of the number of common factors.

An important stage in the estimation of the model is the adequate selection of the number of common factors, $r$. Here we illustrate the application of the method developed by Forni et al. (2000) for this purpose.

The spectral density matrix is computed for a grid of frequencies. Then, the so called "dynamic eigenvalues" are computed by calculating the eigenvalues of the upper-left $m_{1}$ by $m_{1}$ submatrices of the complete $m$ by $m$ spectral density matrices. In Fig. 8 the averages over frequencies considered of these eigenvalues are depicted. The horizontal axis corresponds to the number of crosssectional units $m_{1}$ (which is maximum when the complete vector of series $m=24$ is considered). The first $r$ dynamic eigenvalues exhibit an approximately constant positive slope, while the remaining ones are flatter. The variance explained by the $r^{\text {th }}$ factor is substantially larger than that explained by the $(r+1)^{\text {th }}$. This procedure is illustrated for the case under study in Fig. 8. At first glance, maybe only one common factor could be considered. But, when inspecting in more detail Fig. 8, the slope for the second common factor is larger than the subsequent eigenvalues (all of them in light blue). For several $m_{1}$ 's the corresponding second eigenvalue doubles the third one and of course all the other ones. For sure, according to Fig. 8, the first

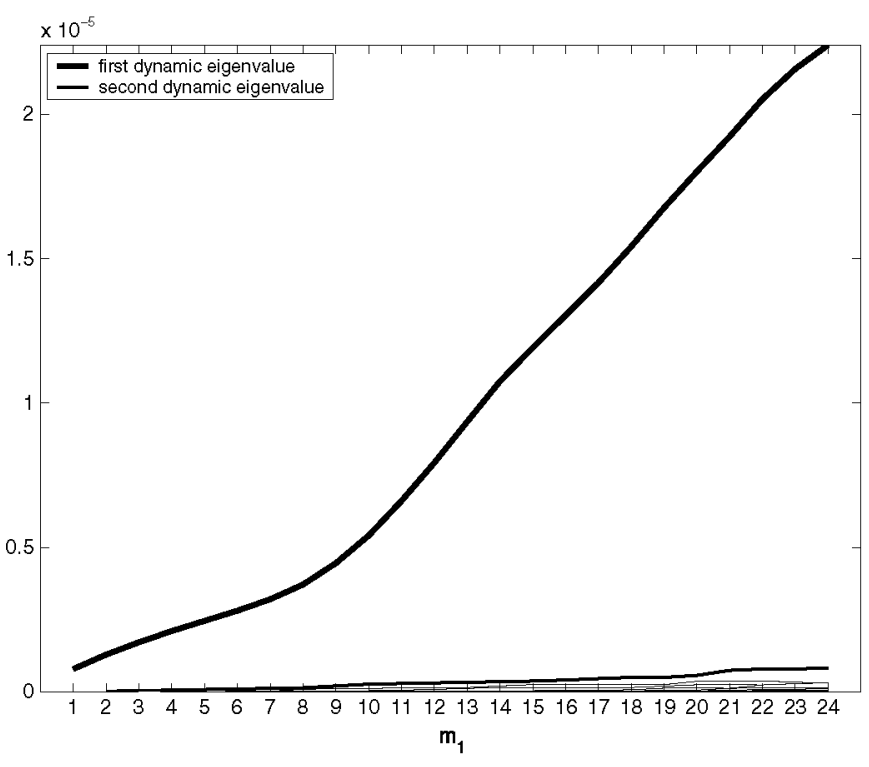

Fig. 8. Testing for the number of common factors.

common factor would explain much more of the total variability than the second one. Particularly, in the case under study, our main objective is to describe the common dynamics in mean and variance sufficiently well and overall, using this model to forecasting, so not so great parsimony is needed. Considering $r=2$ common factors means a great dimension reduction, since the original data were 24-dimensional. Finally, in this sense it is also relevant that a huge amount of data is used to estimate the model $(24 \times 365 \times 10=87600$ hourly prices). Furthermore, as it is pointed out in Forni et al. (2000), setting a number $r^{*}$ of common factors larger than the true one, $r^{*}$, cannot have dramatic consequences on estimation.

\section{References}

Alonso, A.M., Garcia-Martos, C., Rodríguez, J., Sảnchez, M.J., 2011. Seasonal dynamic factor analysis and bootstrap inference: application to electricity market forecasting. Technometrics 53 (2), 137-151.

Ansley, C.F., Kohn, R., 1986. Estimation prediction and interpolation for ARIMA models with missing data. Journal of the American Statistical Association 81 (395), $751-761$

Bollerslev, T., 1986. Generalized autoregressive conditional heteroskedasticity. Journal of Econometrics 31, 307-327.

Bowden, N., Payne, J.E., 2008. Short term forecasting of electricity prices for MISO hubs evidence from ARIMA-EGARCH models. Energy Economics 30, 3186-3197.

Bunn, D.W., 2004. Modelling Prices in Competitive Electricity Markets. Wiley, Chichester (UK).

Bunn, D.W., Karakatsani, N., 2003. Forecasting Electricity Prices. London Business School, London.

Conejo, A.J., Nogales, F.J., Arroyo, J.M., 2002. Price-taker bidding strategy under price uncertainty. IEEE Transactions on Power Systems 17 (4), 1081-1088.

Conejo, A.J., Nogales, F.J., Arroyo, J.M., García-Bertrand, R., 2004. Risk constrained self scheduling of a thermal power producer. IEEE Transactions on Power Systems 19 (3), 1569-1574.

Conejo, A.J., Plazas, M.A., Espinola, R., Molina, A.B., 2005. Day-ahead electricity price forecasting using the wavelet transform and ARIMA models. IEEE Transactions on Power Systems 20 (2), 1035-1042.

Conejo, A.J., Carrión, M., Morales, J.M., Nogales, J., 2010. Electricity pool prices: longterm uncertainty characterization for futures-market trading and risk management. Journal of the Operational Research Society 61, 235-245.

Connor, G., Korajczyk, R., Linton, O.B., 2006. The common and specific components of dynamic volatility. Journal of Econometrics 132 (1), 231-255.

Contreras, J., Espínola, R., Nogales, F.J., Conejo, A.J., 2003. ARIMA models to predict nextday electricity prices. IEEE Transactions on Power Systems 18 (3), 1014-1020.

Diebold, F.X., Nerlove, M., 1989. The dynamics of exchange rate volatility: a multivariate latent factor ARCH model. Journal of Applied Econometrics 4 (1), 1-21.

Durbin, J., Koopman, S.j., 2001. Time series analysis by state space methods. Oxford University Press.

Escribano, A., Peña, J.I., Villaplana, P., 2002. Modelling Electricity Prices: Internationa Evidence. Working Paper 02-27, Economic Series, Universidad Carlos III de Madrid.

Engle, R.F., 1982. Autoregressive conditional heteroscedasticity with estimates of the variance of United Kingdom inflation. Econometrica 50 (4), 987-1007. 
Engle, R.F., 1987. Multivariate ARCH with Factor Structures-Cointegration in Variance. University of California Press.

Forni, M., Hallin, M., Lippi, M., Reichlin, L., 2000. The generalized dynamic-factor model identification and estimation. The Review of Economics and Statistics 82 (4), $540-554$.

Garcỉa-Ferrer, A., Gonzâlez-Prieto, E., Peña, D., 2011. A conditionally heteroskedastic independent factor model with an application to financial stock returns. International Joumal of Forecasting 33 (6), 1227-1239.

Garcia-Martos, C., Rodríguez, J., Sánchez, M.J., 2007. Mixed models for short-run forecasting of electricity prices: application for the Spanish market. IEEE Transactions on Power Systems 2 (2), 544-552.

García-Martos, C., Rodríguez, J. and Sánchez, M.J. forthcoming. "Short-term Forecasting of Electricity Prices Using Mixed Models," Advances in Electric Power and Energy: Price and Load Forecasting in Electric Power Systems, Power Systems Engineering, Power Engineering Series of IEEE Press/Wiley books, Wiley.

Geweke, J., Singleton, K.J., 1981. Maximum likelihood 'confirmatory' factor analysis of economic time series. International Economic Review 22, 37-54.

Harvey, A.C., 1989. Forecasting Structural Time Series Models and the Kalman Filter Cambridge University Press, Cambridge.

Harvey, A., Ruiz, E., Sentana, E., 1992. Unobserved component time series models with ARCH disturbances. Journal of Econometrics 52, 129-158.

Higgs, H., 2009. Modelling price and volatility inter-relationships in the Australian wholesale spot electricity markets. Energy Economics 31 (5), 748-756.

Higgs, H., Worthington, A., 2008. Stochastic price modelling of high volatility, meanreverting, spike-prone commodities: the Australian wholesale spot electricity market. Energy Economics $30(6), 3172-3185$.

Huisman, R., Huurman, C., Mahieu, R., 2007. Hourly electricity prices in day-ahead markets. Energy Economics 29, 240-248.

King, M., Sentana, E., Wadhwani, S., 1994. Volatility and links between national stock markets. Econometrica 62 (4), 901-933.

Knittel, C.R., Roberts, M.R., 2005. An empirical examination of restructured electricity prices. Energy Economics 27, 791-817.
Koopman, S.J., Ooms, M., Carnero, M.A., 2007. Periodic seasonal Reg-ARFIMA-GARCH models for daily electricity spot prices. Journal of the American Satistical Association 102 (477), 16-27.

Lee, R.D., Carter, L.R., 1992. Modelling and forecasting U.S. mortality. Journal of the American Statistical Association 87 (419), 659-671.

Lucia, J.J., Schwattz, E.S., 2002. Electricity prices and power derivatives: evidence from the Nordic power exchange. Review of Derivatives Research 5, 5-50.

Nogales, F.J., Contreras, J., Conejo, A.J., Espinola, R., 2002. Forecasting next-day electricity prices by time series models. IEEE Transactions on Power Systems 17 (2), 342-348.

Ortega, J.A., Poncela, P., 2005. Joint forecasts of Southern European fertility rates with nonstationary dynamic factor models. International Journal of Forecasting 21, 539-550.

Peña, D., Box, G.E.P., 1987. Identifying a simplifying structure in time series. Journal of the American Statistical Association 82 (399), 836-843.

Peña, D., Poncela, P., 2004. Forecasting with nonstationary dynamic factor models. Journal of Econometrics 119, 291-321.

Peña, D., Poncela, P., 2006. Nonstationary dynamic factor analysis. Journal of Statistical Planning and Inference 136, 1237-1257.

Rodríguez, A.F., Ruiz, E., 2009. Bootstrap prediction intervals in state space models. Journal of Time Series Analysis 30 (2), 167-178.

Smith, M., Cottet, R., 2006. Estimation of a longitudinal multivariate stochastic volatility model for the analysis of intra-day electricity prices. Working Paper ECMT2006-1, Sydney University, School of Economics and Political Science.

Stock, J.H., Watson, M., 2002. Forecasting using principal components from a large number of predictors. Journal of the American Statistical Association 97, 1167-1179.

Trapero, J.R., Pedregal, D., 2009. Frequency domain methods applied to forecasting electricity markets. Energy Economics 31, 727-735

Tsay, R., 2005. Analysis of Financial Time Series. Wiley Press.

Vehvilainen, I., Pyykkonen, T., 2005. Stochastic factor model for electricity spot price: the case of the Nordic market. Energy Economics 27, 351-367. 